\title{
Analysis of Unsteady Wave Processes in a Rotating Channel
}

L.M. Larosiliere

Ohio Aerospace Institute

Brook Park, Ohio

and

M. Mawid

University of Illinois at Chicago

Chicago, Illinois

Prepared for the

29th Joint Propulsion Conference and Exhibit

cosponsored by the AIAA, SAE, ASME, and ASEE

Monterey, California, June 28-30, 1993 
$\cdots \cdot$

.

yol
y 


\title{
ANALYSIS OF UNSTEADY WAVE PROCESSES IN A ROTATING CHANNEL
}

\author{
L.M. Larosiliere \\ Ohio Aerospace Institute \\ Lewis Research Center \\ Cleveland, Ohio 44135 \\ and \\ M. Mawid \\ University of Illinois at Chicago \\ Chicago, Illinois 60680
}

\begin{abstract}
The impact of passage rotation on the gasdynamic wave processes is analyzed through a numerical simulation of ideal shock-tube fiow in a closed rotating-channel. Initial conditions are prescribed by assuming homentropic solid-body rotation. Relevant parameters of the problem such as wheel Mach number, hub-to-tip radius ratio, length-to-tip radius ratio, diaphragm temperature ratio, and diaphragm pressure ratio are varied. The results suggest possible criteria for assessing the consequences of passage rotation on the wave processes, and they may therefore be applicable to pressure-exchange wave rotors. It is shown that for a fixed geometry and initial conditions, the contact interface acquires a distorted three-dimensional timedependent orientation at non-zero wheel Mach numbers. At a fixed, wheel Mach number, the level of distortion depends primarily on the density ratio across the interface as well as the hub-to-tip radlus ratio. Rarefaction fronts, shocks, and contact interfaces are observed to propagate faster with increasing wheel Mach number.
\end{abstract}

\section{NOMENCLATURE}

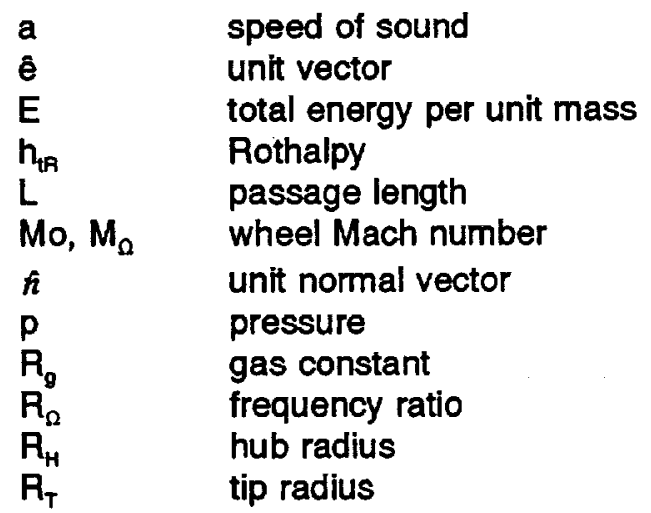

entropy per unit mass

temperature

time

relative velocity vector

ratio of specific heats

density

passage sector angle

rotational speed

dyadic vector product

Subscripts

L

left side (high pressure) of diaphragm in shock-tube flow equilibrium state for initial conditions right side (low pressure) of diaphragm in shock-tube flow uniform reference state axis of rotation center of rotation

\section{INTRODUCTION}

The goal of this paper is to analyze, for shock-tube flow, the manner in which centripetal and Coriolis accelerations brought on by the rotation of a closed channel impact the wave processes. Wave processes considered are gasdynamic ones, including shock waves, contact interfaces, and rarefaction waves, but excluding deflagrations and detonations. Herein, shock-tube flow is generated by the instantaneous rupture of a diaphragm separating a high pressure gas from a lower pressure gas of the same composition. Gasdynamic waves are initiated and propagated in a closed rotating-channel. Apart from general interest in unsteady compressible flow, understanding the propagation and interaction of gasdynamic waves in a rotating channel is useful for pressure-exchange wave rotor [1] applications. For a wave rotor, prediction of wave propagation speeds as well as distortion of fluid 
contact interfaces are required for performance assessment.

The approach taken is to formulate the rotating shock-tube problem in terms of the threedimensional unsteady Euler equations in a rotating reference frame. Dimensionless variables are introduced thereby allowing relevant parameters to be identified. Our aim is to characterize the influence of these parameters on the wave processes. Although we are primarily concerned with the unsteady gasdynamics context, we are careful to vary the parameters of the problem in a range applicable to wave rotor designs.

The one-dimensional theory of shock-tube flow in a closed stationary channel is reviewed by Glass and Patterson [2]. A classical shock-tube consists of a channel initially separated in two parts by a diaphragm. Both parts are filled with gas, which is permitted to settle to rest and a uniform state so that its temperature becomes uniform throughout the tube, but the pressure in the two parts are arranged to be different. When the diaphragm is instantaneously ruptured (in the ideal case), a wave system consisting of a shock followed by a fluid contact interface is transmitted into the low pressure side of the channel and a centered rarefaction front propagates into the high pressure side. This wave system can be represented in an $(x, t)$ plane as shown in Figure 1. The left $(L)$ and right $(R)$ states are prescribed at time $t=0$ and the waves are illustrated with straight lines emanating from the origin. Note that the rarefaction front consists of a series of Mach lines along which flow quantities are constant but vary from line to line. State (2), which is compressed by the shock wave, and state (3), which is formed from the isentropic expansion of the gas in the high pressure side through the rarefaction front, are uniform regions. The fluid velocity and the pressure in the two uniform states are identical. However, because of the different formative processes involved, the temperature, density, and entropy of the two states are different. Hence, these two uniform states are separated by a contact interface. For a perfect gas, the wave speeds and the properties of the uniform states are readily determined from the initial conditions.

For the case of a rotating shock-tube, the centripetal and Coriolis accelerations may disrupt the one-dimensionality of the classical shock-tube flow. To the authors' knowledge, the question of exactly how the wave processes differ from the classical non-rotating shock-tube for a given rotational speed, has not been addressed in the open literature. The primary aim of this paper is to provide the correct framework in which to answer this question and to give specific numerical examples illustrating this framework.

This paper is organized as follows. In the next section, the equations of motion, written in a rotating reference frame, are made dimensionless. This results in a clear identification of the relevant parameters of the problem. In the following section, numerical solutions of the governing equations for various ranges of the problem parameters are presented and discussed. These results suggest criteria for estimating the extent to which passage rotation impacts the gasdynamic wave processes. Finally, a summary of the major findings is given.

\section{FORMULATION}

The geometric configuration chosen to illustrate the effects of passage rotation consists of a closed cylindrical-channel (Fig. 2) rotating about its length. Viscous and heat conduction contributions are neglected. This reduces the mathematical formulation to that of the Euler equations in a rotating reference frame.

The rotating shock-tube problem involves a combination of the classic stationary shock-tube flow in a closed channel and the homentropic flow of a fluid undergoing solid-body rotation. Boundary and initial conditions for these two flows may be anticipated to define reference scales of the problem. The flow is initiated by first imposing homentropic solid-body rotation on the fluid on both sides of the diaphragm and then rupturing the diaphragm thereby generating the wave system. Note that the uniform entropy of the gas is different on each side of the diaphragm. Of particular interest here, is the orientation of the contact interface at specific positions along the length of the closed channel. Thus, the relevant time scale is the time for waves to propagate axially along the channel. This time scale is on the order of $L / a_{-}$. The tip radius is taken to be the length scale over which centrifugal effects are important while the length 
of the channel is assumed to correspond to the wave propagation length scale.

\section{Governing Equations}

Dimensionless variables are introduced in order to reduce the number of parameters that occur explicitly. Consistent with the problem at hand, the equations are made dimensionless by scaling with the channel length $L$ for the axial dimension, the tip radius $R_{T}$ for the radial dimension, a density $\rho_{\infty}$ a speed of sound $a_{\text {oos }}$ and a reference time $L / a_{-}$. The uniform thermodynamic reference state " $\infty$ ", is that which would exist at the center of rotation in the low pressure side of the passage prior to rupturing the diaphragm if the height of the channel were to be extended to the center of rotation.

Combinations of $L, R_{T}, \rho_{\infty}$, and $a_{\infty}$ are selected so that the following variables, defined without asterisks, are dimensionless:

$$
\begin{gathered}
x=\frac{x^{*}}{L}, t=\frac{t^{*}}{L a_{\infty}}, \rho=\frac{\rho^{*}}{\rho_{-}}, \vec{w}=\frac{\vec{w}^{*}}{a_{\infty}}, p=\frac{p^{*}}{\rho_{-} a_{-}^{2}}, \\
R=\frac{R^{*}}{R_{T}}, E=\frac{E^{*}}{a_{\infty}^{2}}, s=\frac{s^{*}}{\gamma R_{g}}, \vec{\nabla}=L \vec{\nabla}^{*} .
\end{gathered}
$$

Let $(R, x, \theta)$ denote cylindrical coordinates attached to the rotating channel. The $x$-axis lies along the axis of rotation and the rotating channel is restricted by the six walls: $\theta=0, \theta=\Phi, R=$ $R_{H} / R_{T}, R=1, x=0$, and $x=1$. The flow is modelled by the unsteady three-dimensional Euler equations [3] written in a rotating reference frame,

$$
\begin{gathered}
\frac{\partial \rho}{\partial t}+\vec{\nabla} \cdot(\rho \vec{w})=0 \\
\frac{\partial \rho \vec{w}}{\partial t}+\vec{\nabla} \cdot(\rho \vec{w} \otimes \vec{w})+ \\
2 R_{\mathrm{Q}} \rho \varepsilon_{\mathrm{Q}} \times \vec{w}-\rho M_{\mathrm{Q}}^{2} \vec{\nabla}\left(\frac{R^{2}}{2}\right)=-\vec{\nabla} p \\
\frac{\partial \rho E}{\partial t}+\vec{\nabla} \cdot\left(\rho \vec{w} h_{t R}\right)=0
\end{gathered}
$$

completed by the perfect gas state relation,

$$
T=\gamma \frac{p}{\rho}
$$

where $h_{\mathrm{TR}}$ is the rothalpy,

$$
h_{2 R}=\frac{T}{(\gamma-1)}+\frac{1}{2} w^{2}-\frac{1}{2} M_{Q}^{2} R^{2},
$$

and $E$ is the total energy per unit mass in the rotating frame,

$$
E=h_{2 R}-\frac{p}{\rho} .
$$

Free-slip wall velocity boundary conditions are:

$$
\vec{w} \cdot \hat{h}=0 \text { for },\left\{\begin{array}{rr}
R=1, & R=\left(\frac{R_{H}}{R_{T}}\right) \\
x=0, & x=1 \\
\theta=0, & \theta=\Phi
\end{array}\right\}(\mathrm{lg})
$$

The dimensionless parameter $R_{R}$ is defined as:

$$
R_{0}=\frac{L / a_{-}}{1 / \Omega}
$$

This parameter is a measure of the ratio of the time scale of motion (i.e., transit time) to that of rotation. It should be noted that $R_{12}$ scales only the Coriolis acceleration and is thus unimportant if this acceleration is not significant. Another dimensionless parameter is the wheel Mach number based on tip rotational speed defined as:

$$
M_{\mathrm{o}}=\frac{\Omega R_{T}}{a_{-}}
$$

This is primarily a measure of the relative strength of the centripetal acceleration. Note that $R_{\Omega}=M_{\Omega} L / R_{T}$.

The governing equations and boundary conditions clearly show the relevant parameters of the problem to be: the wheel Mach number $M_{0}$, the length-to-tip radius ratio $L / R_{T}$, the hub-totip radius ratio $R_{H} / R_{T}$, the passage sector angle $\Phi$, and the initial conditions. 
Initial Conditions

Steady homentropic $(\vec{\nabla} s=0)$, solid-body rotation $(\vec{w}=0)$ is assumed to exist in both sides of the diaphragm prior to it being ruptured. Note that the entropy differs on each side of the diaphragm. Under these conditions, and using the Gibbs relation,

$$
T \vec{\nabla} s=\frac{\vec{\nabla} T}{\gamma-1}-\frac{\vec{\nabla} p}{\rho},
$$

the equations of motion can be manipulated (see Vavra [3] for details) to obtain,

$$
\vec{\nabla} h_{R R}=0
$$

Thus, the rothalpy is uniform throughout each side of the diaphragm. The initial states on each side of the diaphragm may be obtained as follows:

$$
\begin{gathered}
T_{o}(R)=T_{C}+\frac{(\gamma-1)}{2} M_{0}^{2} R^{2} \\
P_{o}(R)=P_{C}\left[\frac{T_{o}}{T_{C}}\right] \frac{\gamma}{\gamma-1} \\
P_{o}(R)=\gamma \frac{P_{o}}{T_{o}}
\end{gathered}
$$

where $P_{c}$, and $T_{c}$ are the pressure and temperature at the center of rotation on each side of the diaphragm (i.e., $P_{L}, T_{L}, P_{A}, T_{R}$ ).

\section{Computational Scheme}

For this investigation, a two-stage RungeKutta scheme developed by Chima and Yokota [4] was applied to solve the Euler equations. This scheme uses explicit time-marching and central difference spatial discretization in a boundaryfitted H-mesh. Gasdynamic discontinuities (i.e., shocks and interfaces) are treated by means of a second difference artificial dissipation. This implies that the Rankine-Hugoniot jumps associated with a shock or interface are continuous and spread out over a number of grid points rather than occurring as discontinuities. The dissipation coefficlent was chosen so that the shock thickness is about five grid points irregardless of mesh size. A small amount of fourth difference artificial dissipation is also employed to suppress nonlinear instabilities. The formal truncation error of this scheme is secondorder in space and first-order in time.

The Riemann problem for the special case of shock-tube flow was solved in a stationary wave rotor passage and the results compared with the one-dimensional exact solution. Initial conditions consisted of pressure and density ratios of 2:1 separating left and right states. Good comparison between the exact and the computed solution is shown in Figure 3. Based on these results, the required spatial and temporal resolutions were determined. A longitudinal spatial resolution of $\triangle \mathrm{x} \leq 0.0066$ and a $C F L=0.5$ were set. Further numerical experimentation demonstrated the ability of this scheme to adequately capture the essential features (i.e., wave speeds and interactions) pertinent to this investigation.

\section{RESULTS AND DISCUSSION}

Five cases are presented to illustrate the impact of rotation on the wave processes for shock-tube flow in a rotating passage. The parameters that are varied for each of the cases are given in Table I. The passage sector angle $\Phi$ was maintained at a constant angle of $4.93^{\circ}$ and the diaphragm was initially placed midway in the passage (i.e., $x=0.5$ ). The range of parameters selected reflects possible operating conditions for proposed wave rotors being considered for small gas-turbine thermodynamiccycle performance augmentation.

A: Varying wheel Mach number.-The effects of varying wheel Mach number on the density field are shown in Figure 4 for wheel Mach numbers of $0.0,0.5,1.0$, and 1.5. For each wheel Mach number, all other parameters are held constant as per Table I. Meridional projections of the shock, rarefaction fronts, and interface are illustrated through density contours at four different moments in time.

Three observations follow from Figure 4. First, the shock, contact interface, and rarefaction fronts travel faster with increasing wheel Mach number due to the higher temperature consistent with the centrifugal force field and initial conditions. Second, for the zero wheel Mach number $($ Mo -0$)$, the rarefaction 
TABLE I.-PARAMETERS OF ROTATING SHOCK-TUBE PROBLEM

\begin{tabular}{|l|c|c|c|c|c|}
\hline \multicolumn{1}{|c|}{ Case } & $\mathrm{P}_{\mathrm{L}} / \mathrm{P}_{\mathrm{R}}$ & $\mathrm{T}_{\mathrm{L}} \boldsymbol{T}_{\mathrm{A}}$ & $\mathrm{R}_{\mathrm{H}} / \mathrm{R}_{\mathrm{T}}$ & $\mathrm{L} / \mathrm{R}_{\mathrm{T}}$ & $\mathrm{M}_{\mathrm{Q}} ;$ Mo \\
\hline A: Rotational speed & 2.0 & 1.0 & 0.934 & 1.5 & $0.0,0.5,1.0,1.5$ \\
\hline B: Pressure ratio & $4.0,2.0$ & 1.0 & 0.934 & 1.5 & 0.5 \\
\hline C: Temperature ratio & 2.0 & $0.5,1.0,2.0,4.0$ & 0.934 & 1.5 & 0.5 \\
\hline D: Hub-tip ratio & 2.0 & $1.0,4.0$ & $0.5,0.934$ & 1.5 & 0.5 \\
\hline E: Length & 2.0 & $1.0,4.0$ & 0.934 & $0.75,1.5$ & 0.5 \\
\hline
\end{tabular}

fronts propagate along lines of radially constant density as in a centered expansion fan, whereas for non-zero wheel Mach numbers, the constant density lines in the expansion fan are oblique with the degree of obliquity varying in time as can be seen in a comparison of the Mo -1.0 condition at $t=0.25$ and $t=0.5$. In addition, a nomal shock with a uniform propagation speed is observed. Third, the largest departure from the non-rotating shock-tube is the evolving distortion of the interface with increasing wheel Mach numbers. The initially radial interface becomes oblique prior to any interaction with the reflected shock or rarefaction fronts due to the generation of baroclinic vorticity $(\vec{\nabla} \rho \times \vec{\nabla} p)$. Interactions of the shock and rarefaction fronts with the interface result in stretching of the interface and at the same time, the vorticity generation is amplified. For wheel Mach numbers greater than or equal to one, a rapidly growing non-planar orientation of the interface is observed after it interacts with the shock and rarefaction fronts. This can be better explained by considering the kinematics of stretching and alignment of fluid elements comprising the interface [5]. The evolution of a fluid element is determined by the local velocity gradient tensor which includes a dilatation term (compression or expansion) and a rotational term (vorticity). The combination of these two actions and the wall boundary conditions are responsible for this interface orientation.

A more quantitative illustration of the propagation of the shock and rarefaction fronts is given in Figure 5. It can be seen that the rarefaction fronts propagate in a one-dimensional fashion in terms of rothalpy and axial velocity fields with the initial condition of homentropic solid-body rotation. The increase in wave propagation speed as wheel Mach number is increased from 0 to 1.5 is seen to be significant.
This may have strong implications for wave rotor port-timing in the case where the compressed gas is drawn out of the channel.

The structure of the interface distortion is presented more clearly in Figure 6 where surface plots of the rothalpy distribution are shown in a meridional plane for the four wheel Mach numbers at a time $t=1.0$ after the reflected shock has interacted with the interface. It can be observed that the rothalpy is radially uniform except along the interface for non-zero wheel Mach numbers. An s-shaped fluid interface with an axially-oriented mid-section appears to be developing for non-zero wheel Mach numbers. The extent of the axially-oriented mid-section increases with wheel Mach number. Surface plots of the rothalpy distribution in an axialcircumferential plane at mid-height are shown in Figure 7 for a time $t=1.0$. The plots reveal the extent of interfacial distortion in this plane. In addition, for wheel Mach numbers of one or above, a noticeable non-planar orientation of the interface exists near the trailing side of the passage.

It appears that the interface seeks a 'stable' orientation when viewed in a meridional plane (Figs. 4(d) and 6) as is exhibited for the Mo $=1.5$ case. Stability here implies that the orientation of the interface is stationary to disturbances such as those caused by centripetal acceleration, Coriolis forces, and interaction with waves.

To complete the general picture, axial velocity distributions in a meridional plane and an axial-circumferential plane are presented in Figure 8 and Figure 9 respectively at time $t=1.0$. A vortical structure is observed at the location of the interface for non-zero wheel Mach numbers. The orientation of the structure is consistent with 
that of the interface (Fig. 6 and 7). A radially uniform axial velocity can be observed away from the interface. Thus, the velocity field is onedimensional everywhere except near the interface where the vortical structure induces tangential, and radial velocities. These radial and tangential flows represent flow kinetic energy which is not available to the mean flow, hence their kinetic energy must be considered a contribution to the inefficiency of the process.

B: Varying pressure ratio.-Contour plots of the density field in a meridional plane are presented in Figure 10 at four time sequences for different diaphragm pressure ratios with all other parameters held constant as in Table I. An additional condition is shown for pressure and temperature ratios of 4.0:1 for comparison. Also included, is a sketch of the density ratio across the interface shortly after rupturing the diaphragm. Increasing the diaphragm pressure ratio from 2.0:1 to 4.0:1 results in a relatively more distorted interface. For the temperature and pressure ratios of 4.0:1 condition, atthough not definitively shown in the figures, the orientation of the interface changes direction after interaction with the reflected rarefaction fronts. Note also, the deceleration of the shock wave at $t=1.0$ for $P_{L} / P_{A}=4.0$ and $T_{L} / T_{A}=1.0$ due to propagation in a region of higher fluid velocity.

C: Varying temperature ratio.- The effect of varying diaphragm temperature ratio at Mo $=0.5$ is illustrated in Figure 11 through density contours in a meridional plane at various moments in time. Also indicated, are the density ratios across the interface shortly after rupturing the diaphragm. It can be observed that for temperature ratios of $4.0: 1$ and $0.5: 1$, the interface is significantly distorted even prior to interaction with the reflected shock. Furthermore, for these same temperature ratios, the interface orientation is very non-planar after interaction with the reflected shock $(t=1.0)$. Thus, large density ratios across the interface result in significant distortion of the interface.

Note also, for a temperature ratio of 4.0:1, the interface commences to rollover after interaction with the rarefaction fronts. This is probably caused by a non-uniform dilatation of the fluid elements along the interface. The exact roles of centripetal and Coriolis accelerations in sustaining this interface configuration are not immediately apparent to the authors. In comparison with case A (Fig. 4) and the temperature ratio of $0.5: 1$ condition, the orientation of the interface does not appear to be seeking a stable configuration for this temperature ratio of 4.0:1 condition. It is apparent that for a range of temperature ratios above 1.0, the interface does not acquire a stable configuration, whereas, for temperature ratios of 1.0 or below, the interface configuration becomes stable.

D: Varying $L \mathbb{R}_{T}$ - Figures 12 and 13 illustrate the effect of reducing $L R_{T}$ through density contours in a meridional plane for two different temperature ratios. Recall that the longitudinal coordinates of the channels are dimensionless in these plots (i.e., $x=0-1$.). A better grid resolution was employed for the longer length channel which is why the discontinuities appear to be more smeared for the shorter length. Note that the reference time scale $\left(L / a_{-}\right)$ is different for these two configurations but the dimensionless times are the same. Thus, this is a direct comparison of the effect of changing the residence time of a fluid element within the passage.

Varying $L / R_{T}$ at constant wheel Mach number changes $R_{n}$ which affects the Coriolis term in the equations of motion. Thus, Reducing $L / R_{T}$ should lessen the degree of skewing of the interface as is evident in Figures 12 and 13. The effects of reducing $L / R_{T}$ are more pronounced at the higher temperature ratio. Note that since the distortion of the interface is scaled by $R_{\alpha}$, it may be possible to infer from Fig. 13(b) the role of Coriolis forces in the stability of the interface. These results suggest that depending on the operating conditions, a shorter length or smaller residence time may be better for an application where compressed fluid is drawn out at the end of the channel.

E: Varying hub-tip ratio. - The effects of reducing $R_{H} / R_{T}$ are shown in Figures 14 and 15 . These figures show the meridional distribution of density for two different temperature ratios. It can be observed that increasing the hub-tip ratio generates a larger radial stratification resulting in a non-planar orientation of the interface after interaction with the reflected shock. Varying the hub-to-tip radius ratio changes the stratification height resulting in a radially non-uniform 
baroclinic vorticity generation. Note that for the higher temperature ratio, the rolling over characteristic of the interface is not evident with the lower hub-to-tip ratio.

\section{CONCLUDING REMARKS}

Some insights into the unsteady wave processes in a closed rotating-channel are presented. Analysis of the governing equations revealed the dominant parameters controlling the problem. Three-dimensional numerical results for ideal shock-tube flow in a closed rotating-channel show the influence of these parameters on the flow.

The following conclusions may be drawn concerning the impact of passage rotation on an ideal shock-tube flow having initial conditions of homentropic solid-body rotation in each side of the diaphragm:

1. The rotation of the passage results in a distorted interface with the magnitude of the distortion depending primarily upon the following parameters:

A-the wheel Mach number $M_{\Omega}$,

B-the hub-tip radius ratio $R_{H} / R_{T}$, and

$C$-the density ratio across the interface.

2. For an application where compressed fluid is drawn out at the end of the channel, it may be beneficial to reduce the length-to-tip radius ratio $L / R_{T}$ of the channel so that the interface distortion due to centripetal and Coriolis accelerations is minimized.

3. The shock is nomal to the flow and propagates in a one-dimensional fashion. However, the rarefaction fronts exhibit a twodimensional propagation in terms of the density (also presssure and temperature) field but in terms of axial velocity and rothalpy fields, the propagation of the rarefaction fronts possesses a one-dimensional character.

4. Increasing the wheel Mach number increases the propagation speed of the shock, rarefaction fronts, and also the contact interface. For initial conditions other than those of homentropic solid-body rotation, the above conclusions may not exactly hold. That is, the propagation of the shock and rarefaction fronts may acquire a three-dimensional character. Nevertheless, it is expected that the speed of propagation will still vary in a similar fashion with wheel Mach number. It should also be noted that viscous effects were neglected and it is possible that they may have an appreciable impact on some of the conclusions drawn here.

Definitive guidelines for the design of wave rotors cannot be given since many conflicting parameters are involved. The distortion of the interface may be viewed as a degradation in the volumetric efficlency of the wave rotor cycle. Variations in the propagation speed of the wave fronts as wheel Mach number is increased have design implications for port timings in wave rotor cycles. The present results may be used to assess the impact of passage rotation on the wave processes.

\section{ACKNOWLEDGEMENTS}

We thank Dr. Larry Bober, Chief of Turbomachinery Technology at NASA LeAC, for suggesting this investigation. We also thank our colleagues Fred Newman and Gerard Welch for many useful discussions and valuable suggestions.

\section{REFERENCES}

1. Shreeve, R.P.; and Mathur, A. (eds.): Proceedings of the 1985 ONR/NAVAIR Wave Rotor Research and Technology Workshop. NPS-67-85-008.2

2. Glass, I.I.; and Patterson, G.W.: A Theoretical and Experimental Study of Shock Tube Flow. J. Aeronaut. Sci., Vol. 22, pp. 73-101, 1955.

3. Vavra, M.H.: Aero-Thermodynamics and Flow in Turbomachines. John Wiley \& Sons, Inc., 1960.

4. Chima, R.V.; and Yokota, J.W.: Numerical Analysis of ThreeDimensional Viscous Internal Flows. AIAA J., Vol. 28, No. 5, pp. 798-806, May 1990.

5. Dresselhaus, E.; and Tabor, M.: The Kinematics of Stretching and Alignment of Material Elements in General Flow Fields. J. Fluid Mech. (1991), Vol. 236, pp. 415-44. 


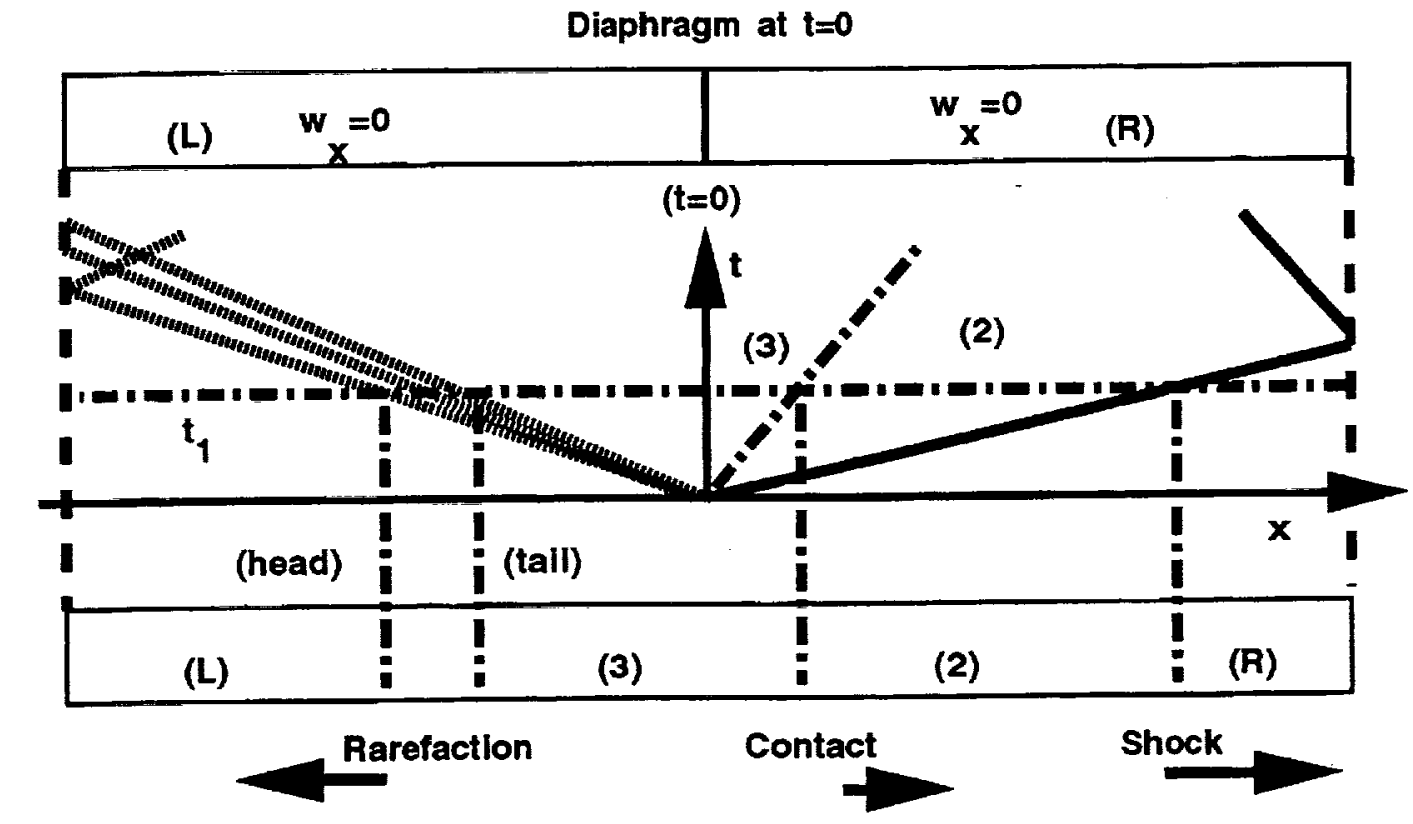

Figure 1. Theoretical $(x-t)$ diagram of the wave system produced in a classical shock-tube flow.

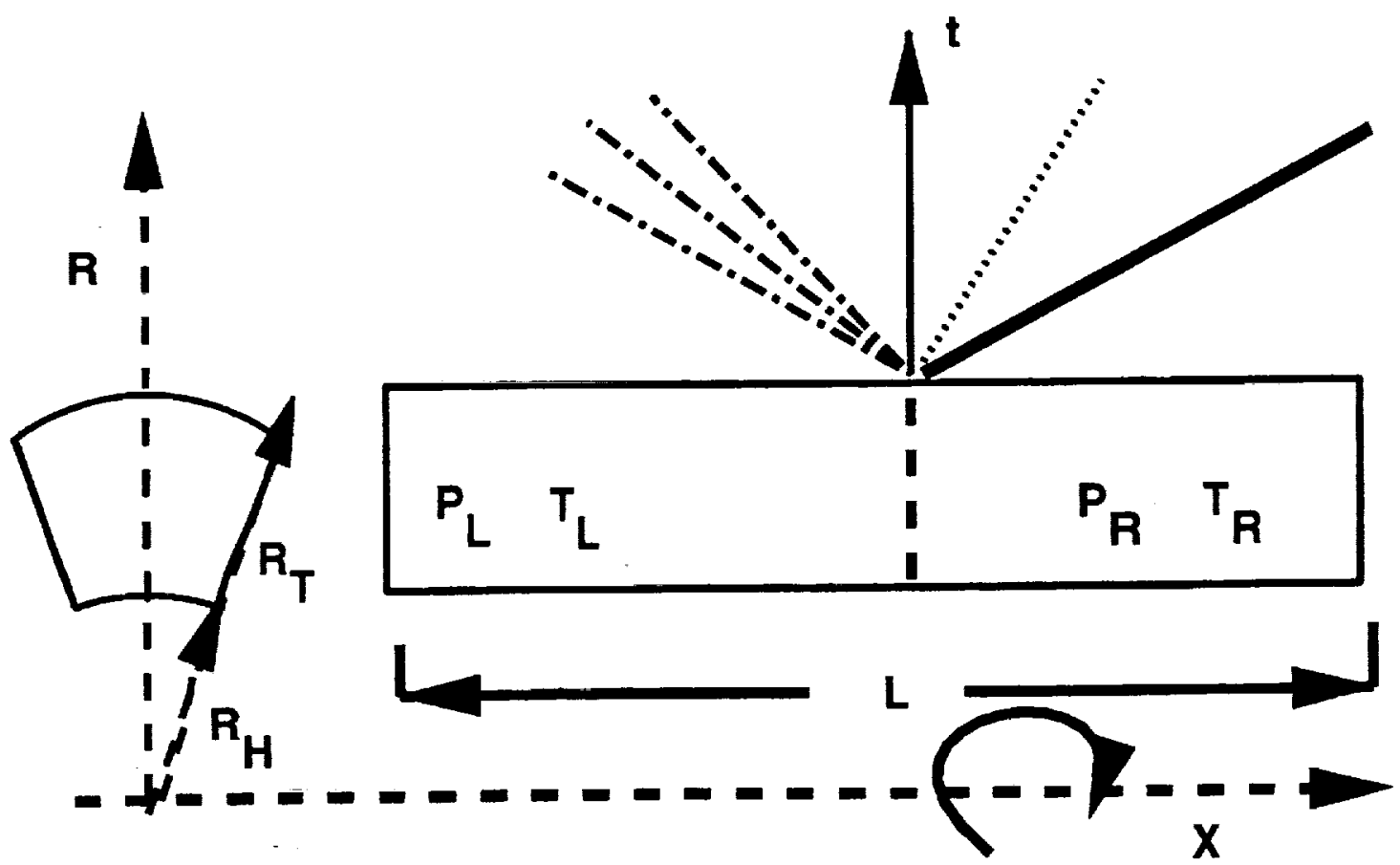

Figure 2. Rotating channel geometry illustrating the generation of a wave system by the instantaneous rupture of a diaphragm. 


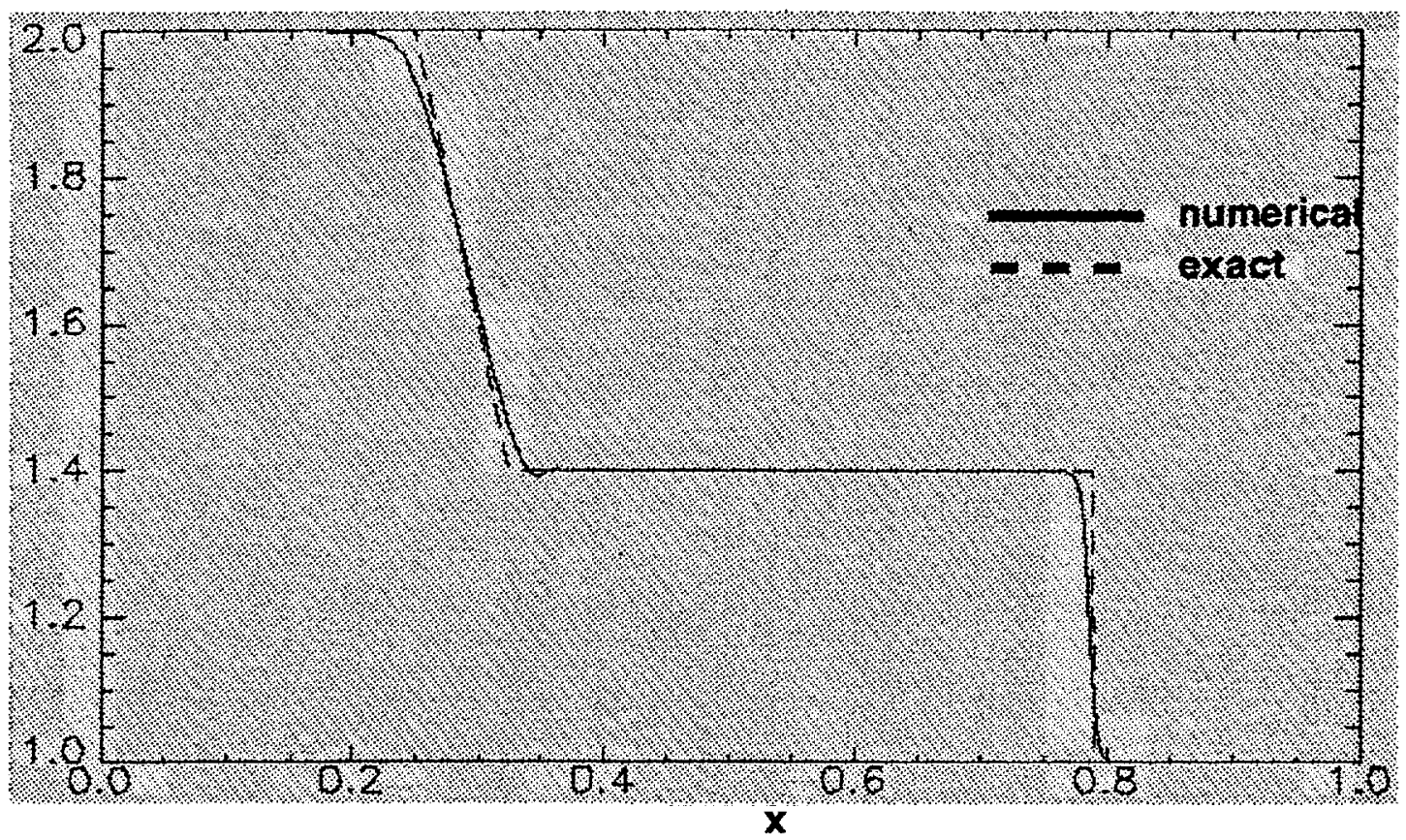

(a) pressure

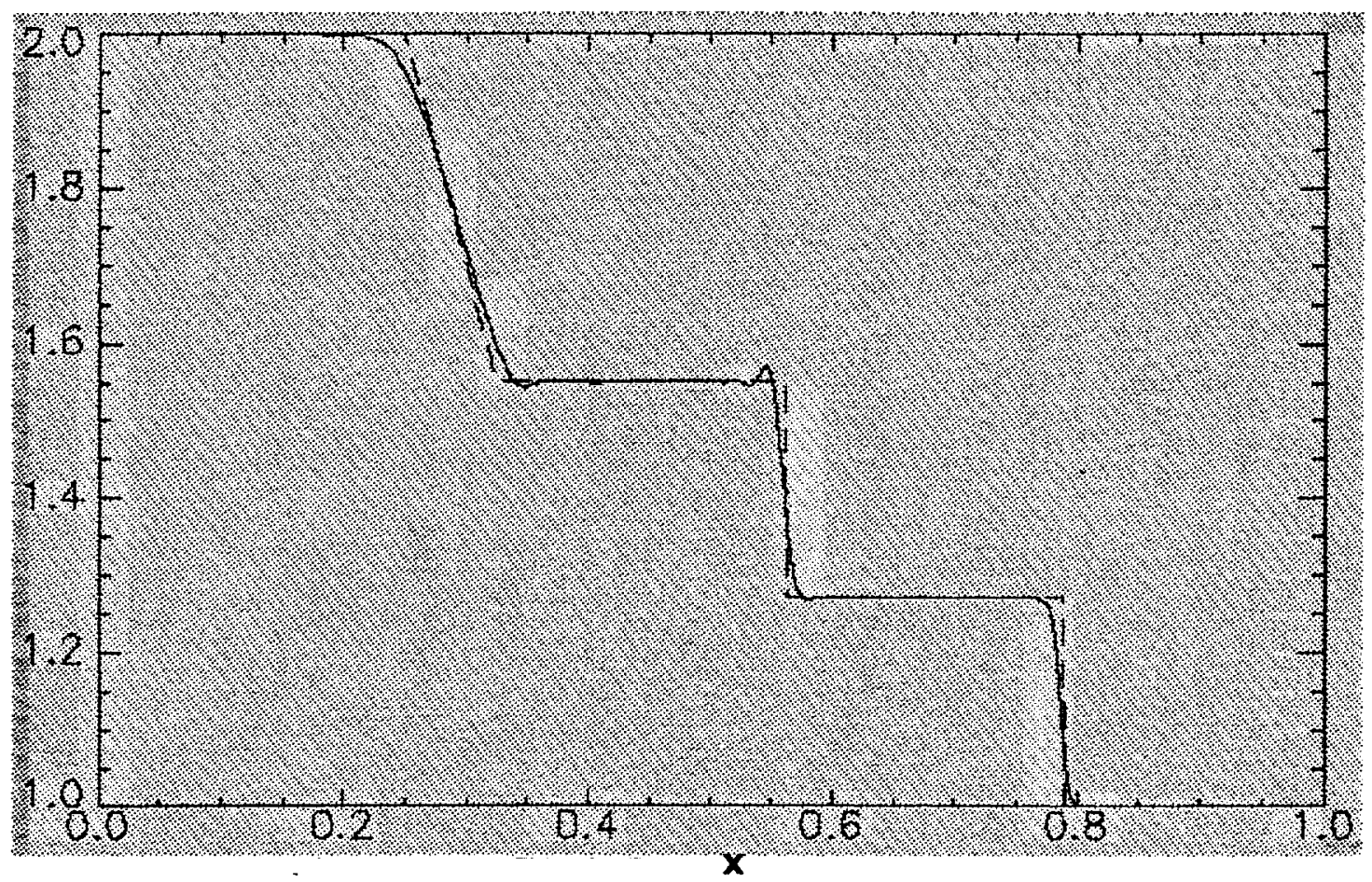

(b) density

Figure 3. Comparison of numerical and exact solutions to the Riemann problem for shock-tube flow. The density and pressure ratios are 2:1 and the time is at $t=0.25$. 


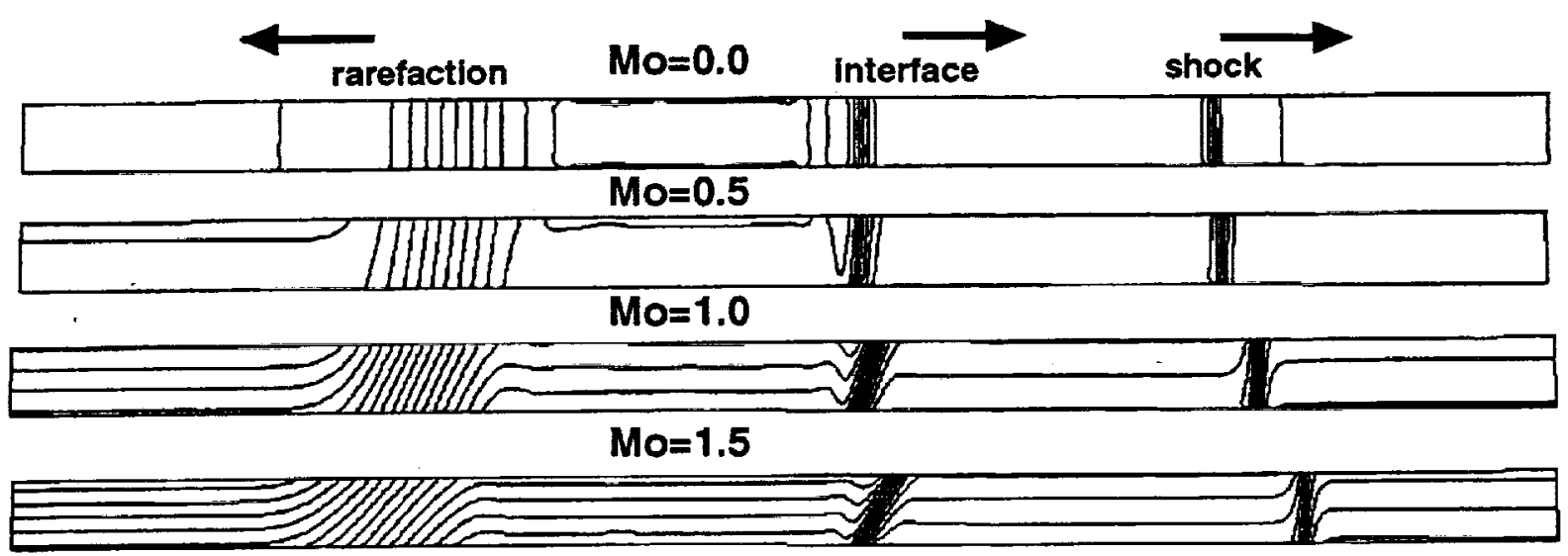

(a) $t=0.25$, shortly after rupturing the diaphragm

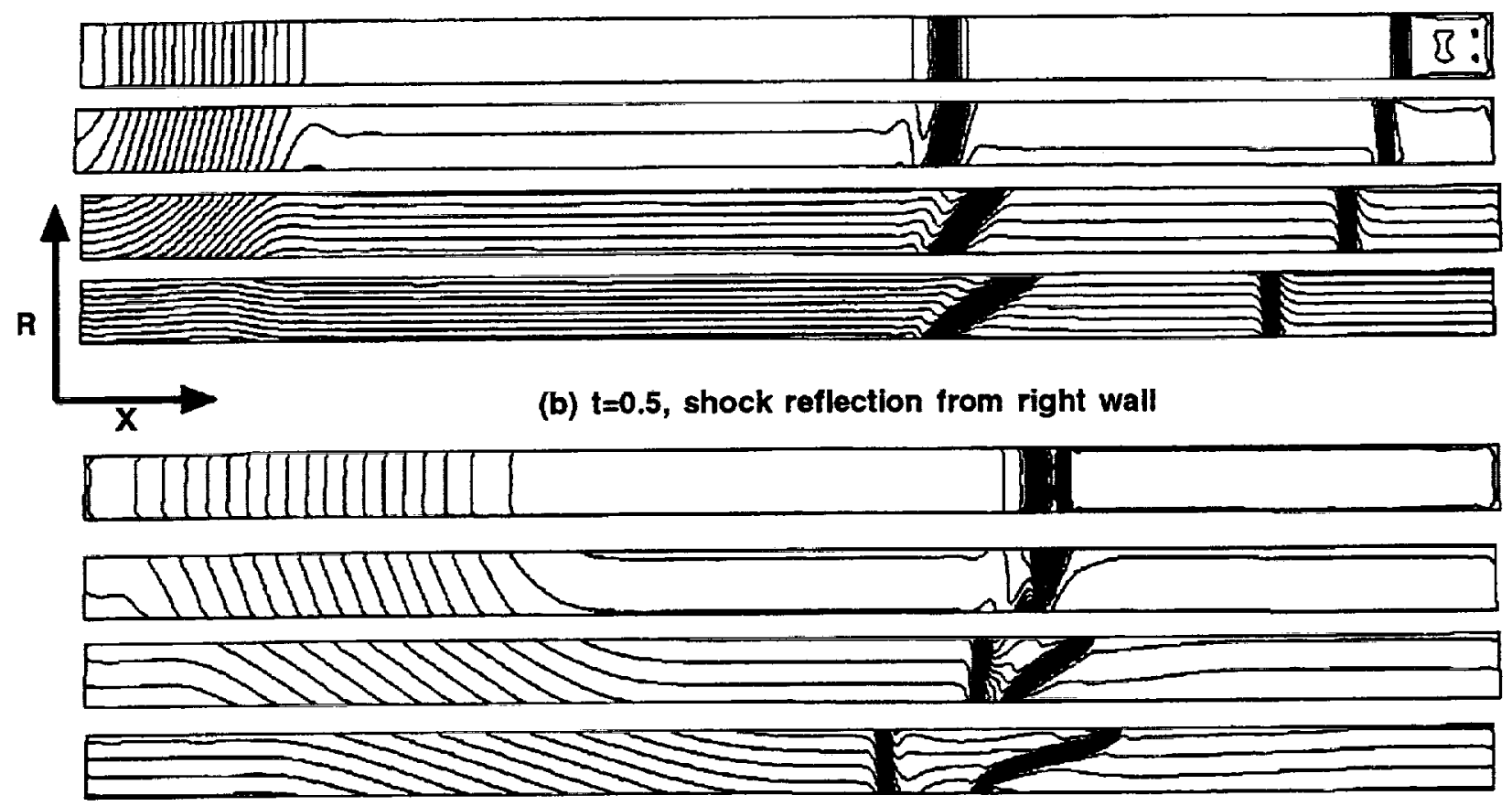

(c) $\mathrm{t}=0.75$, reflected-shock interaction with Interface

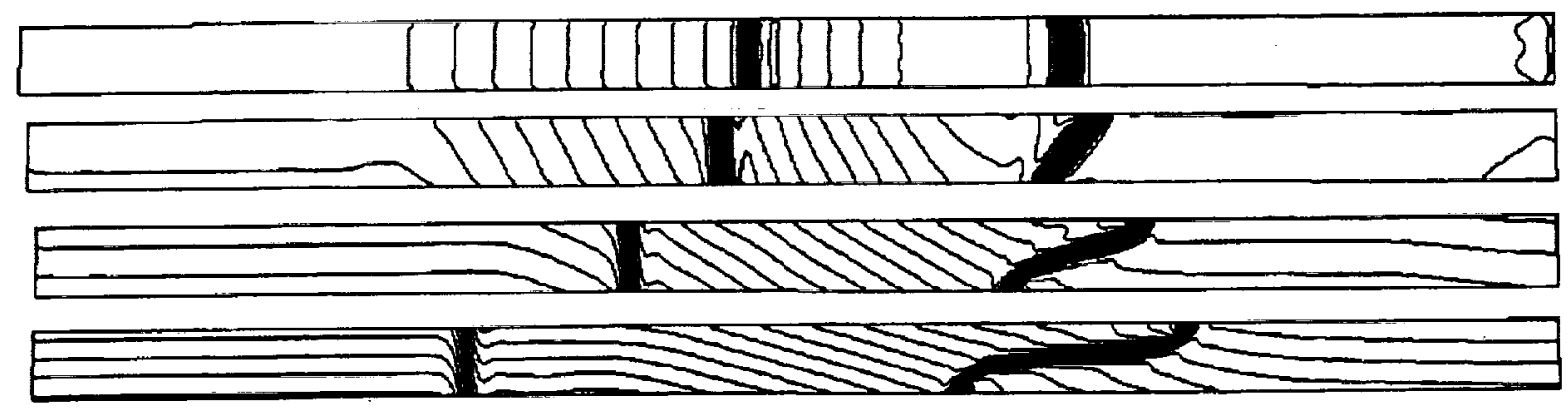

(d) $t=1.0$, reflected-rarefaction front interaction with the shock and interface

Figure 4. Four time sequences showing density contours in a meridional plane for different wheel Mach numbers and other parameters given in Table I for case $A$. The pressure ratio is $2: 1$ and the temperature ratio is $1: 1$. 


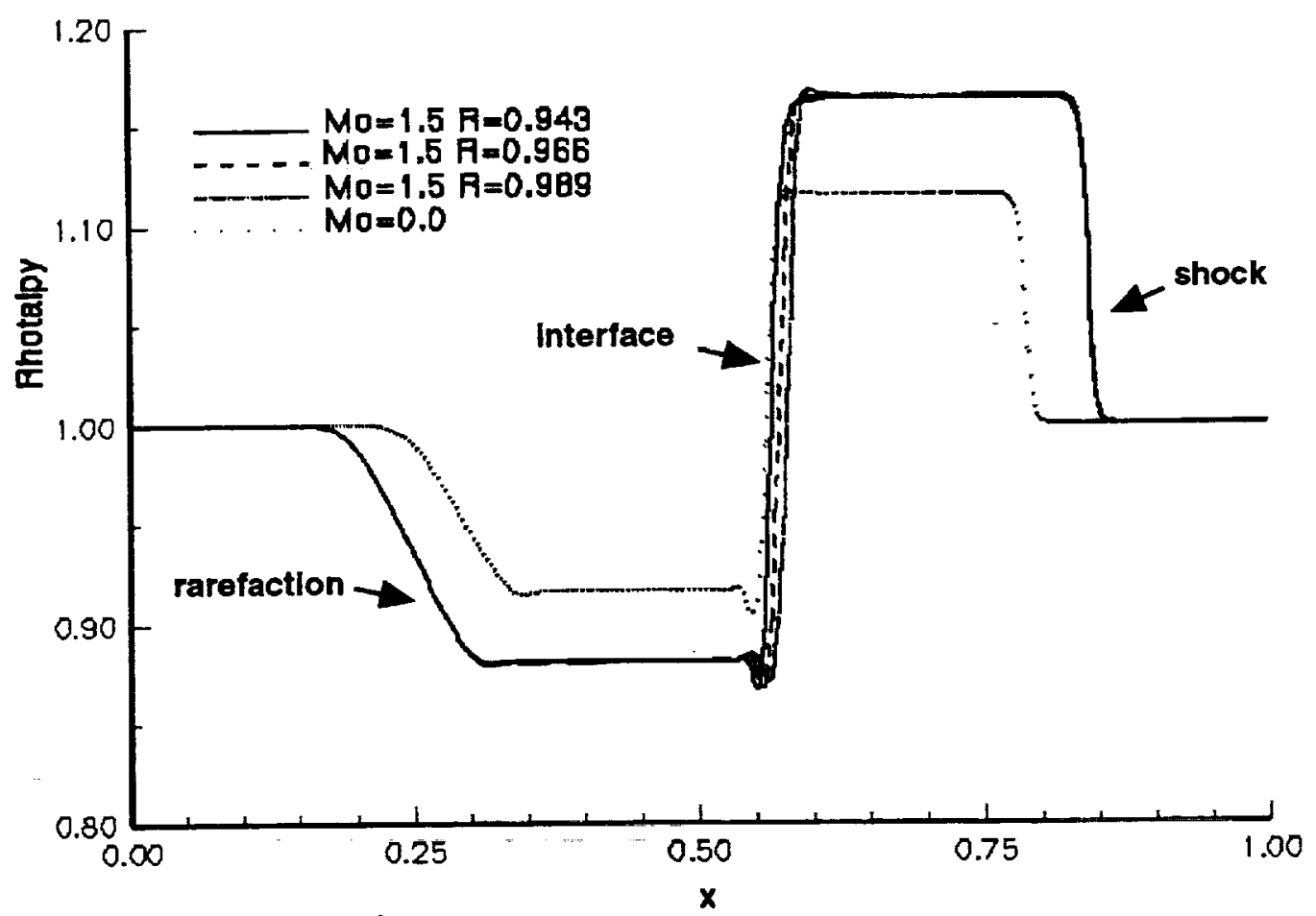

(a) temperature equivalent of rhotalpy

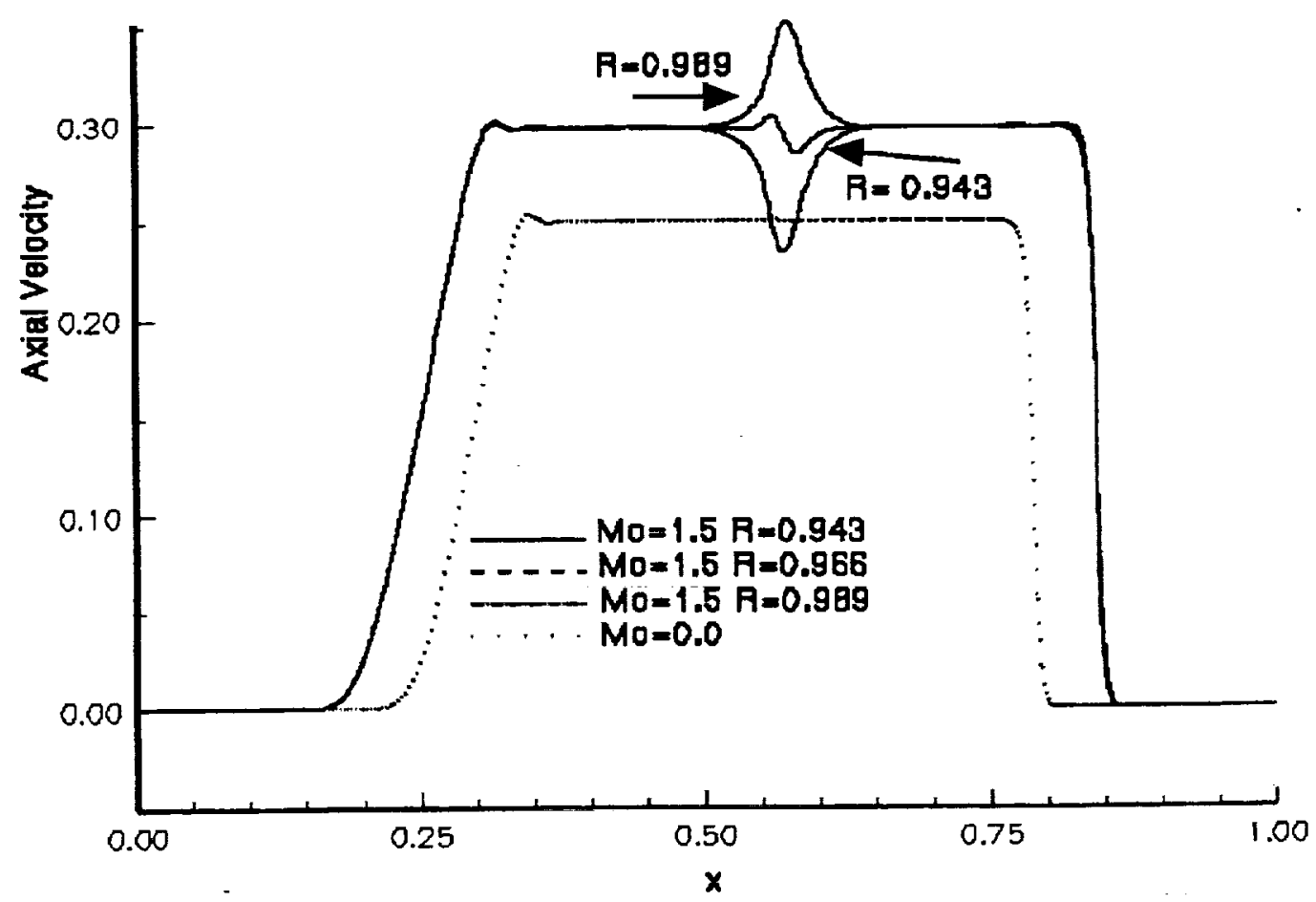

(b) axial velocity

Figure 5. Axial variation of the rhotalpy and axial velocity at $t=0.25$ for wheel Mach numbers of 0.0 and 1.5 and other parameters pertaining to case $A$. For $M O=1.5$, the hub, mean, and tip profiles are also shown. The pressure ratio is $2: 1$ and the temperature ratio is $1: 1$. 

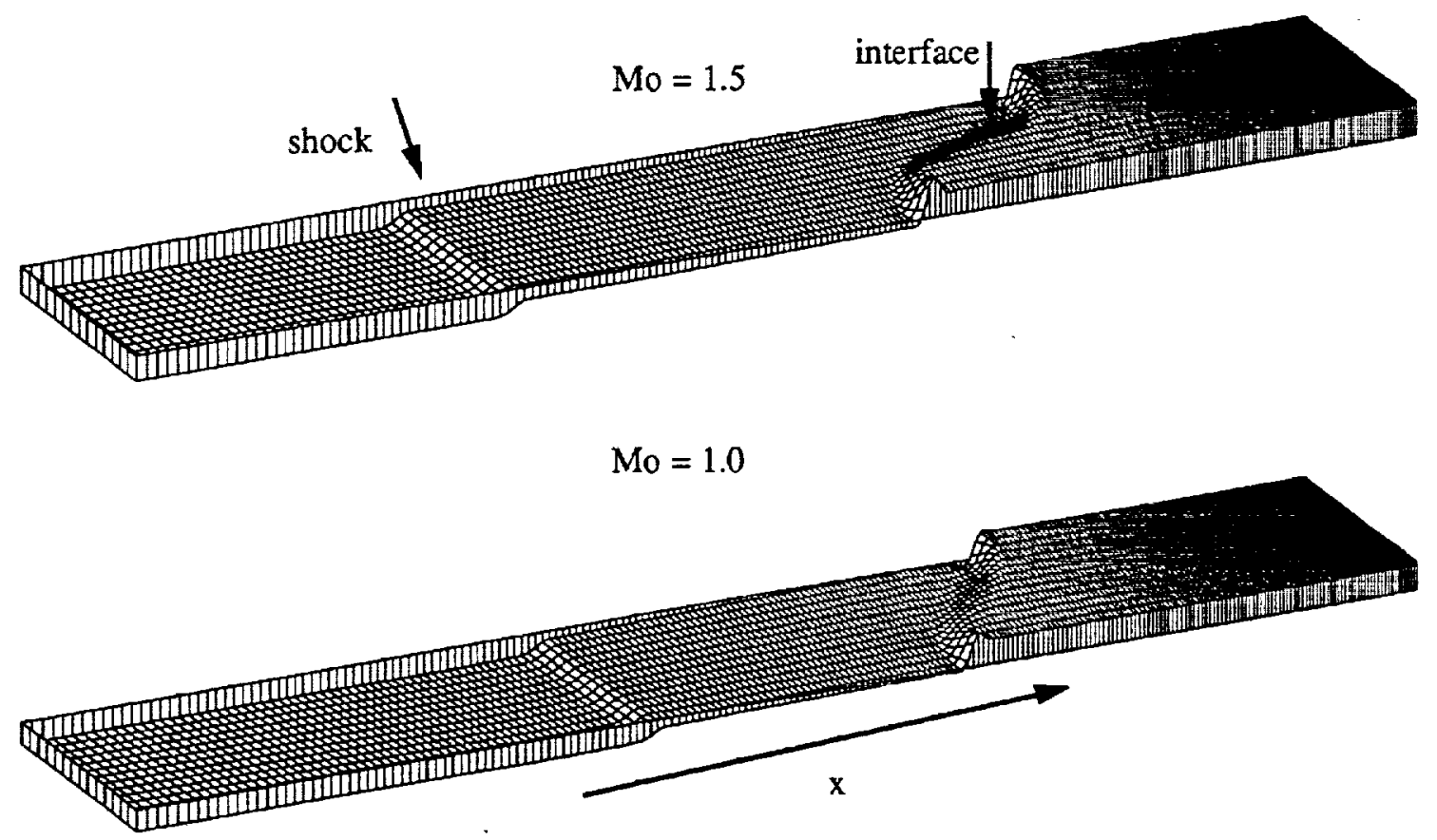

$$
\text { Mo }=0.5
$$
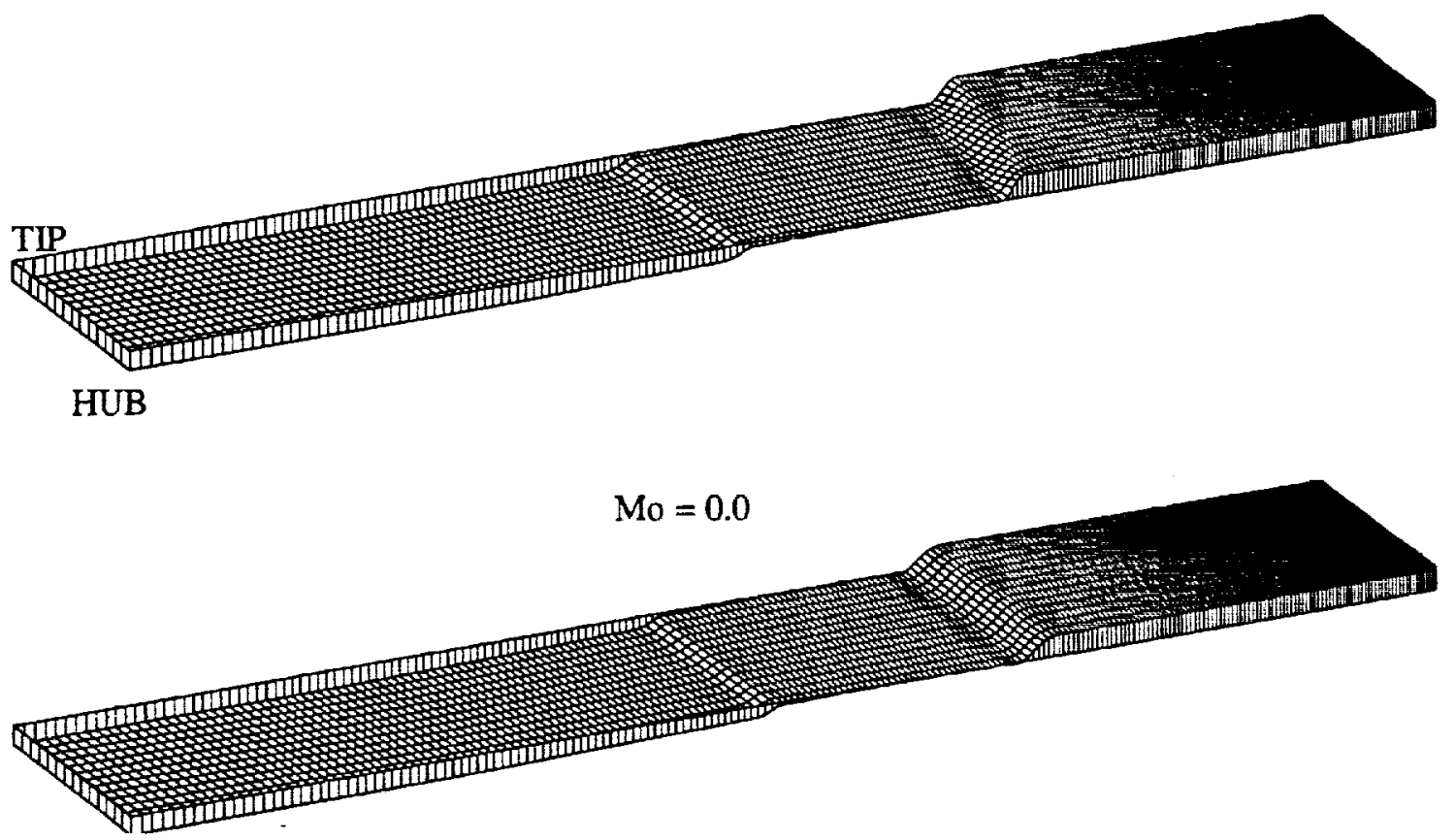

Figure 6. Meridional distribution of rothalpy in mid-passage at $t=1.0$ for various wheel Mach numbers. The pressure ratio is $2: 1$ and the temperature ratio is $1: 1$ (case A). 

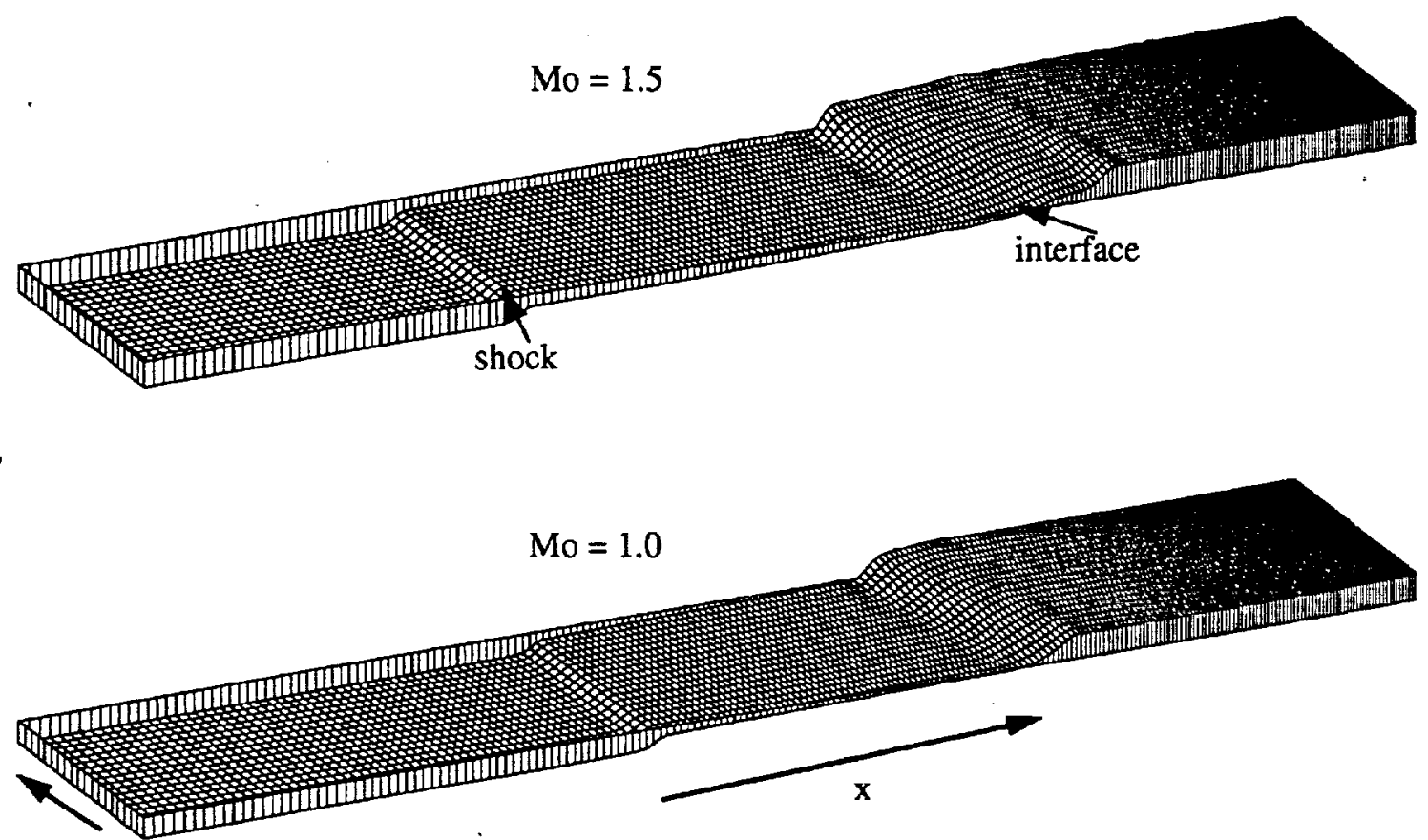

theta

(direction of rotation)
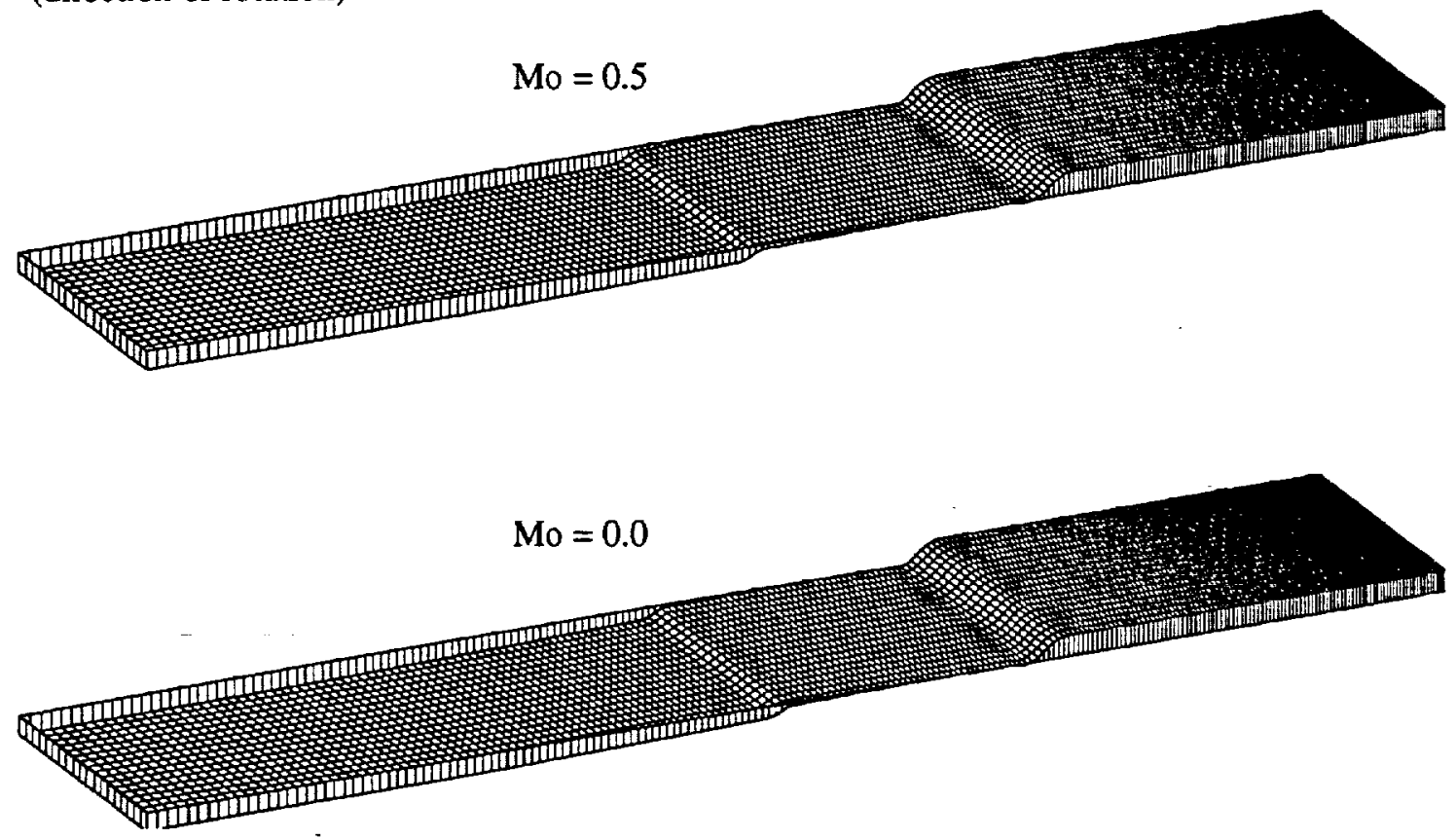

Figure 7. Rhotalpy distribution in an axial-circumferential plane at mid-height for $t=1.0$. The pressure ratio is 2:1 and the temperature ratio 1:1. 

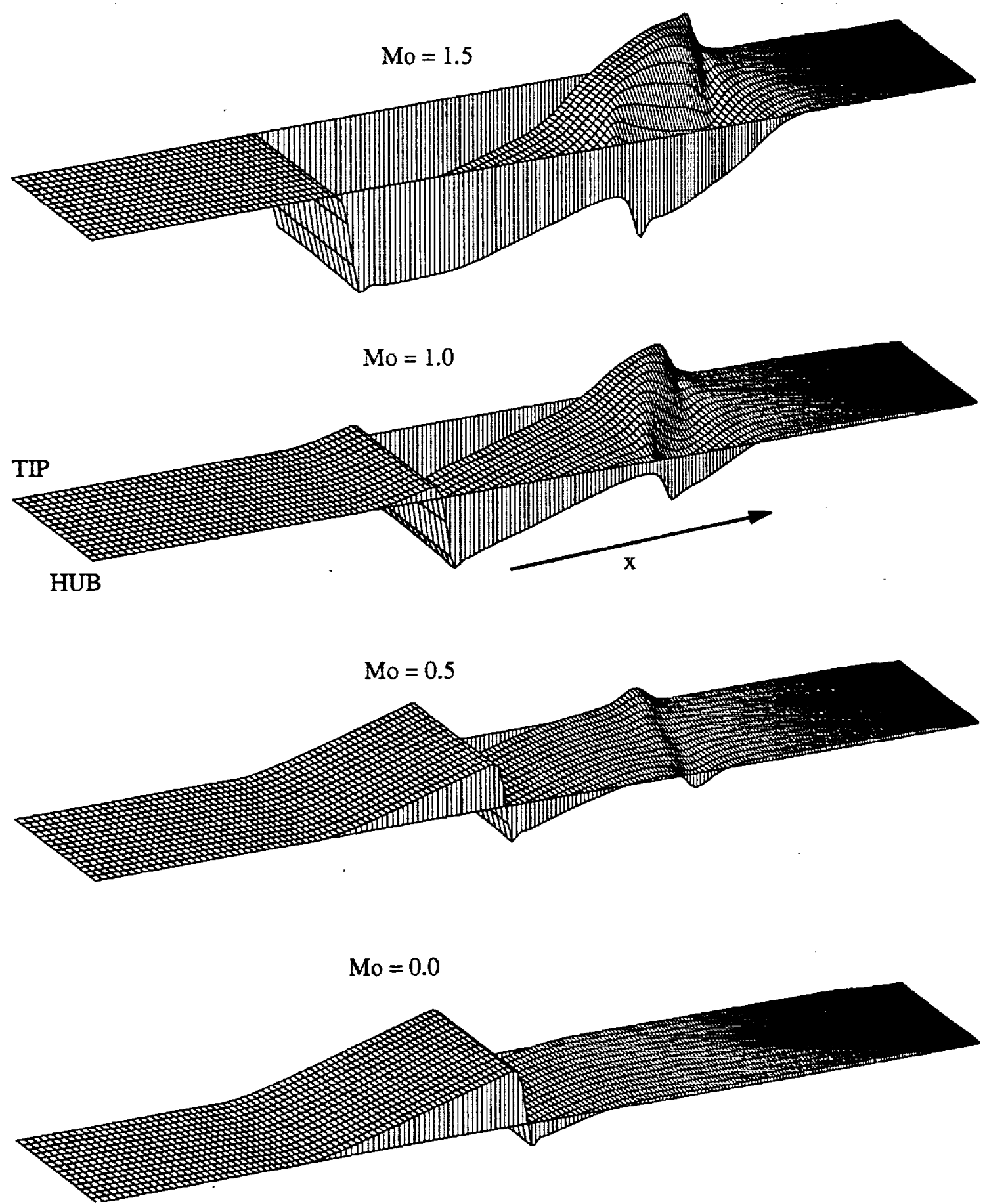

Figure 8. Meridional distribution of axial velocity at $t=1.0$ for various wheel Mach numbers(Mo). The pressure ratio is $2: 1$ and the temperature ratio is $1: 1$ (case A). 

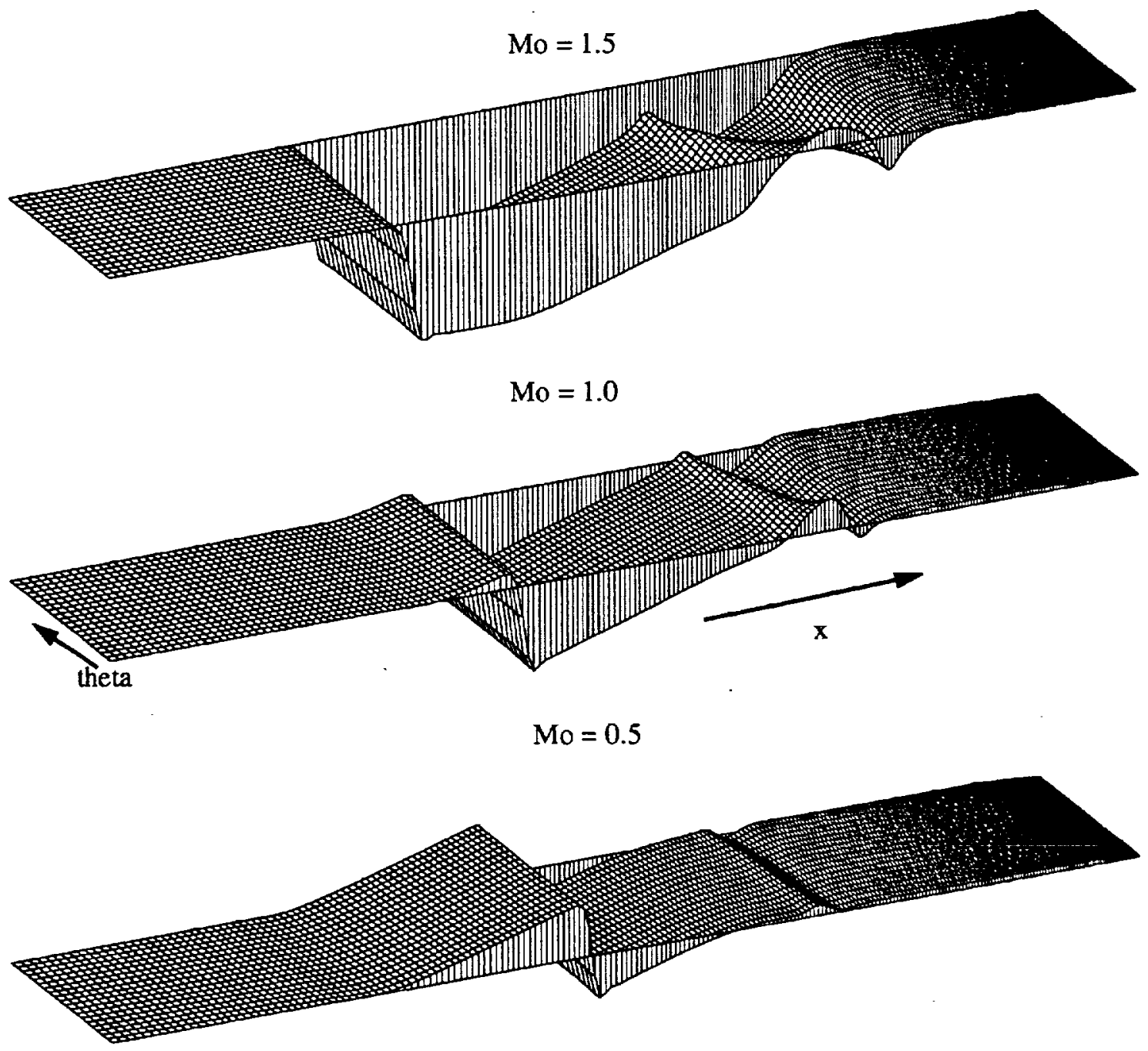

$$
\mathrm{Mo}=0.0
$$

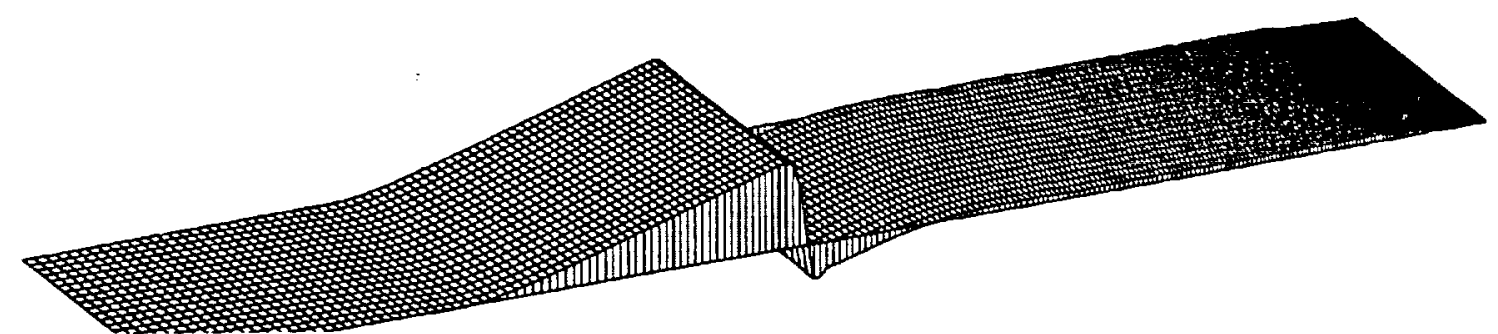

Figure 9. Axial velocity distribution in an axial-circumferential plane at mid-height for $t=1.0$. The pressure ratio is $2: 1$ and the temperature ratio is $1: 1$ (case $\mathrm{A}$ ). 


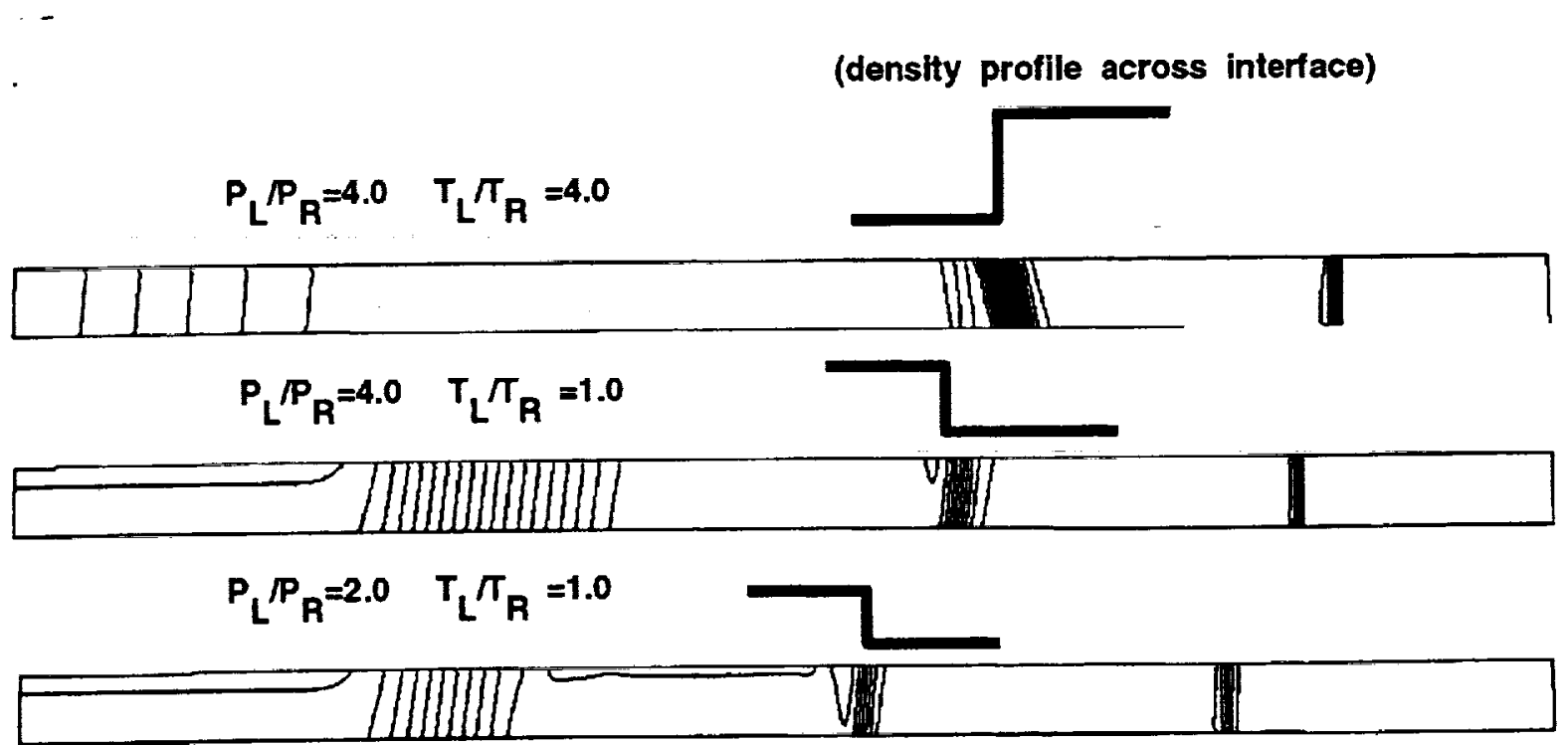

(a) $t=0.25$

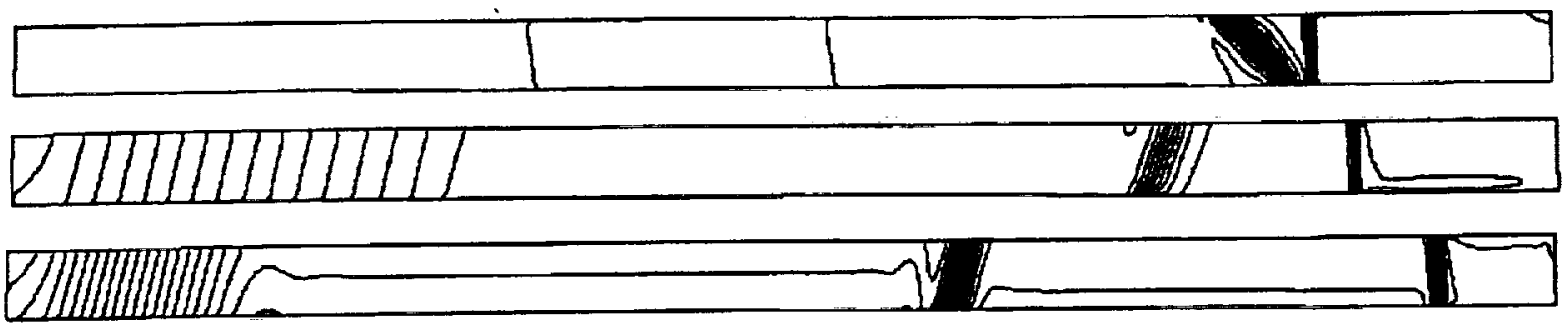

(b) $t=0.50$

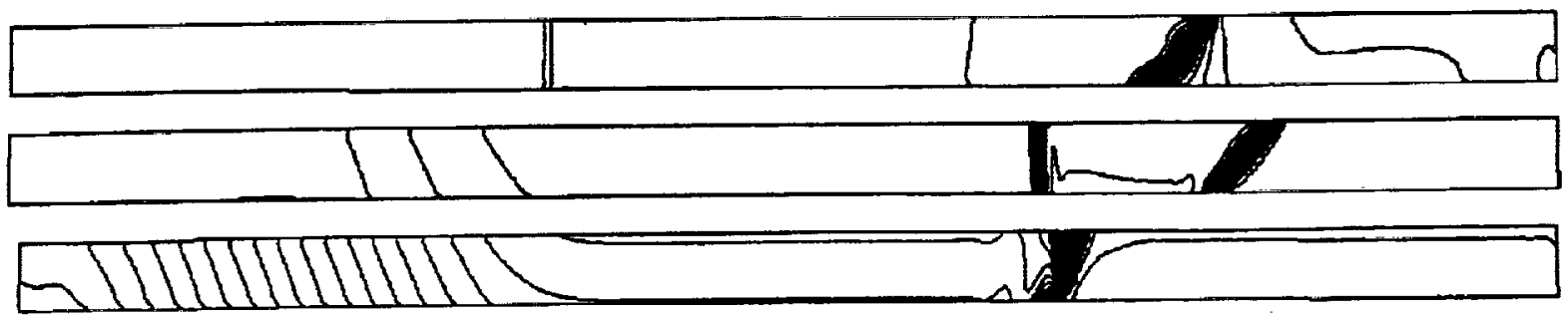

(c) $t=0.75$

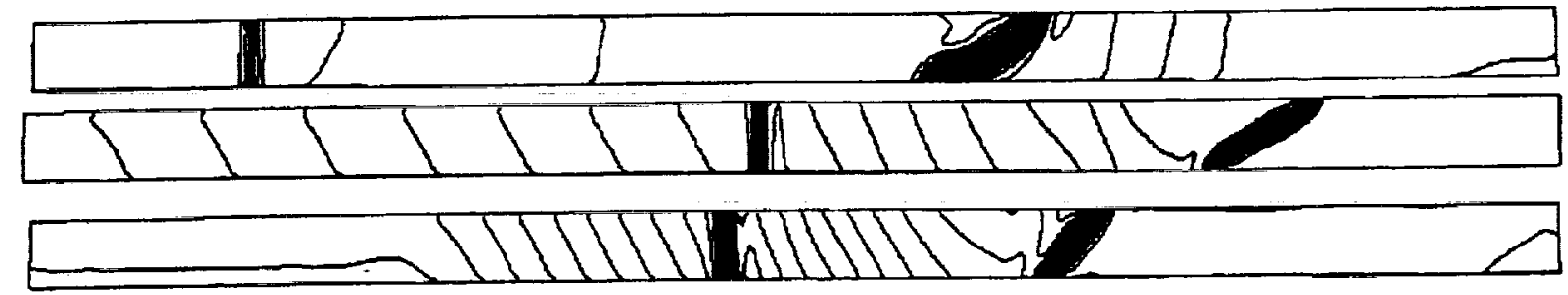

(d) $t=1.0$

Figure 10. Density contours in a meridional plane for four time sequences illustrating the effect of varying diaphragm pressure ratio (case $B$ ). The wheel Mach number is $M 0=0.5$. 


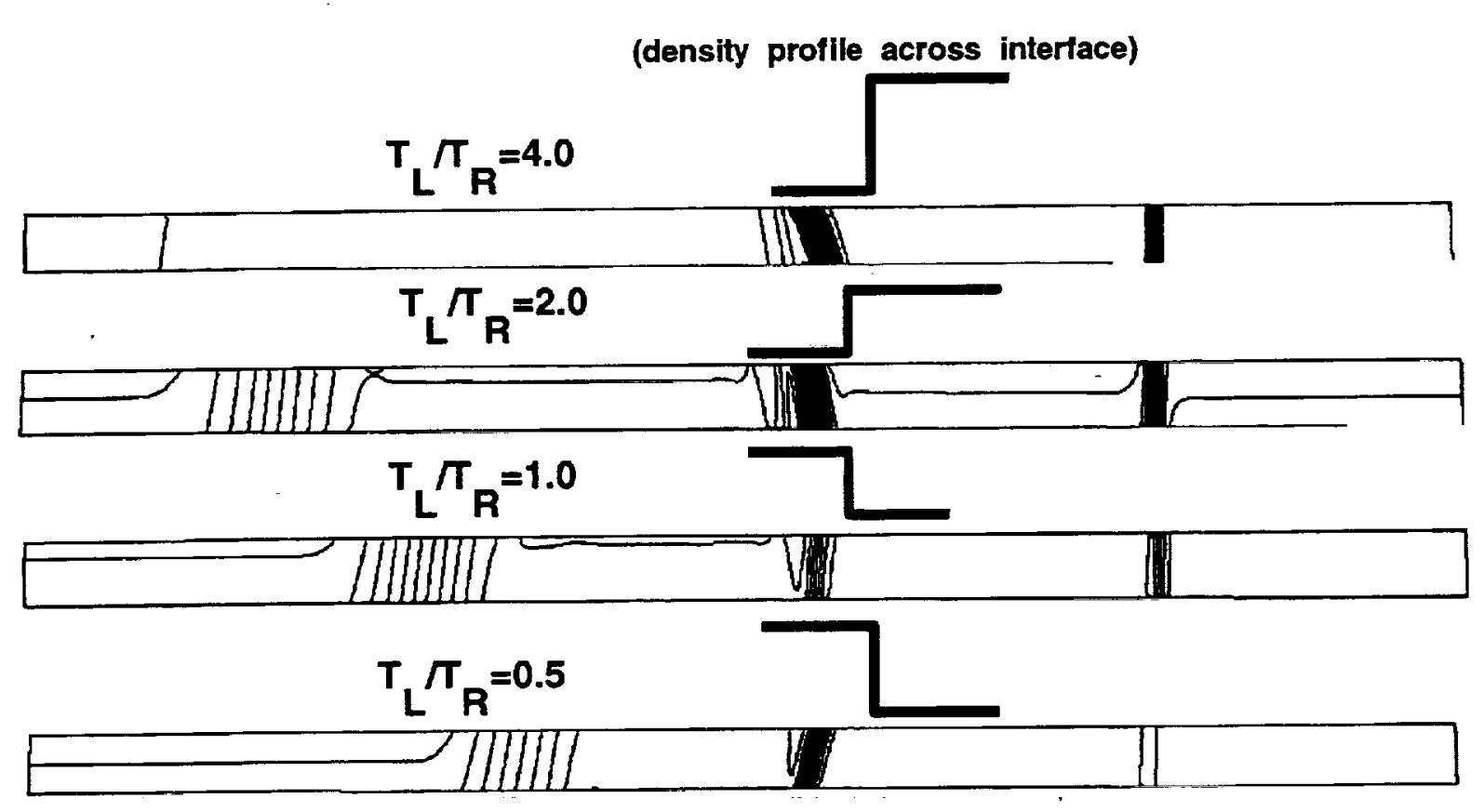

(a) $\mathrm{t}=0.25$

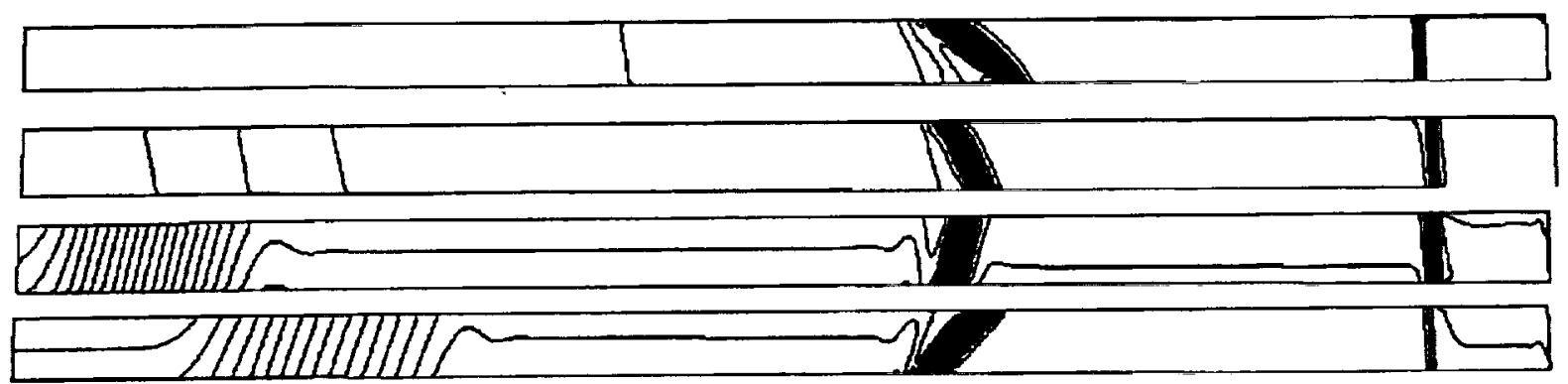

(b) $t=0.5$

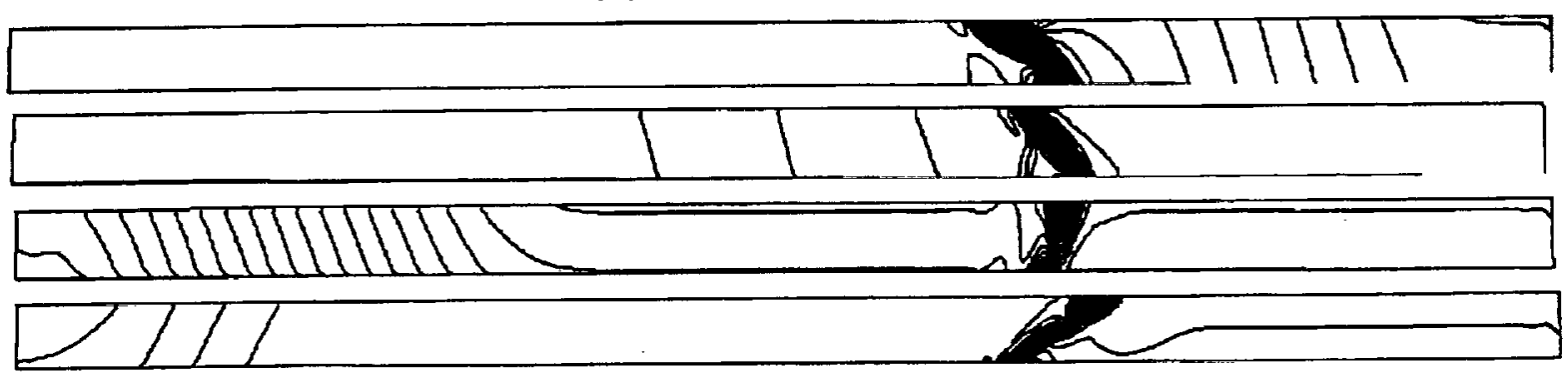

(c) $t=0.75$

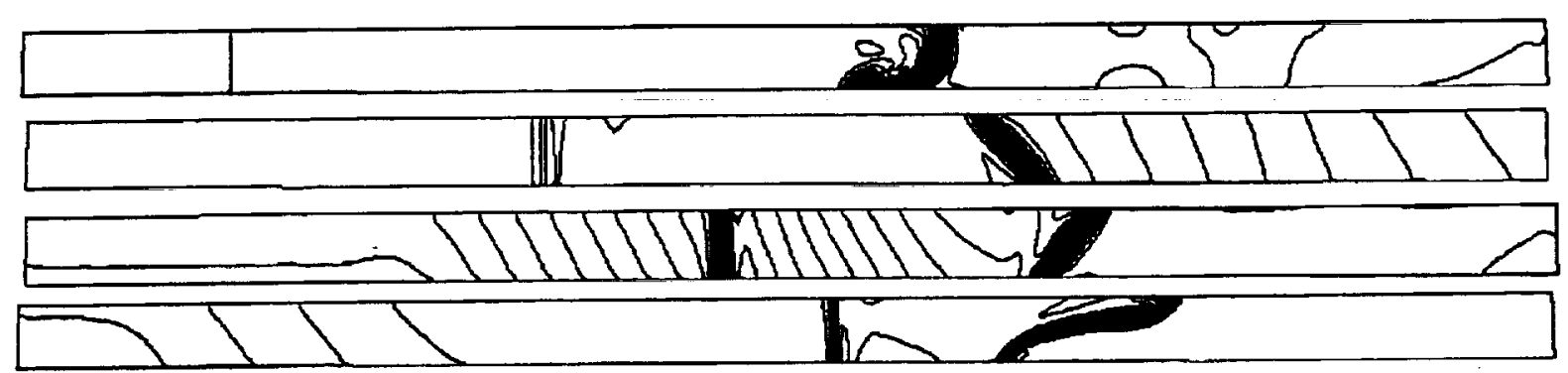

(d) $t=1.0$

Figure 11. Density contours in a meridional plane for four time sequences illustrating the effect of varying diaphragm temperature ratio (case $C$ ). The pressure ratio is $2: 1$ and Mo=0.5. 


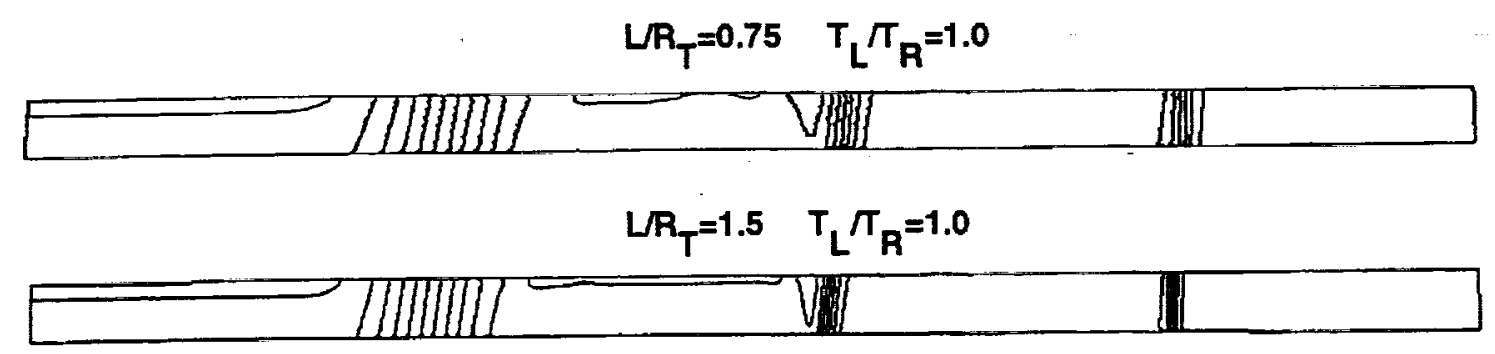

(a) $t=0.25$
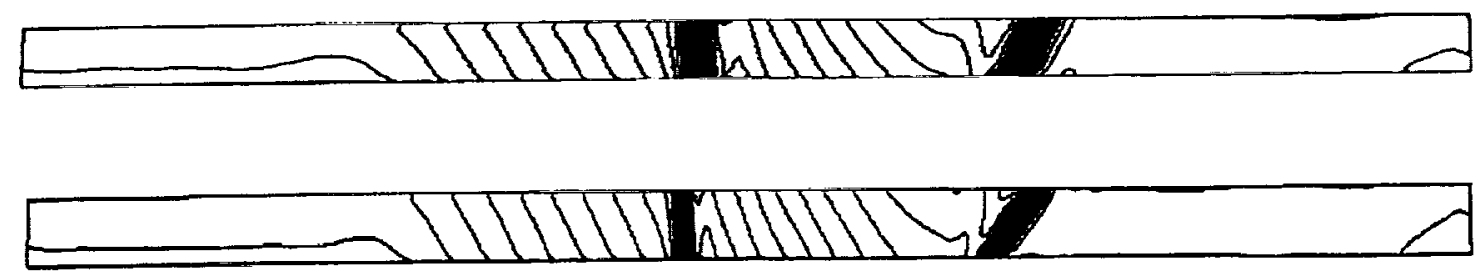

(b) $t=1.0$

Figure 12. Effect of varying length-to-tip radius ratio for $T_{L} / \pi_{R}=1.0$ and $P_{L} / P_{R}=2.0$.

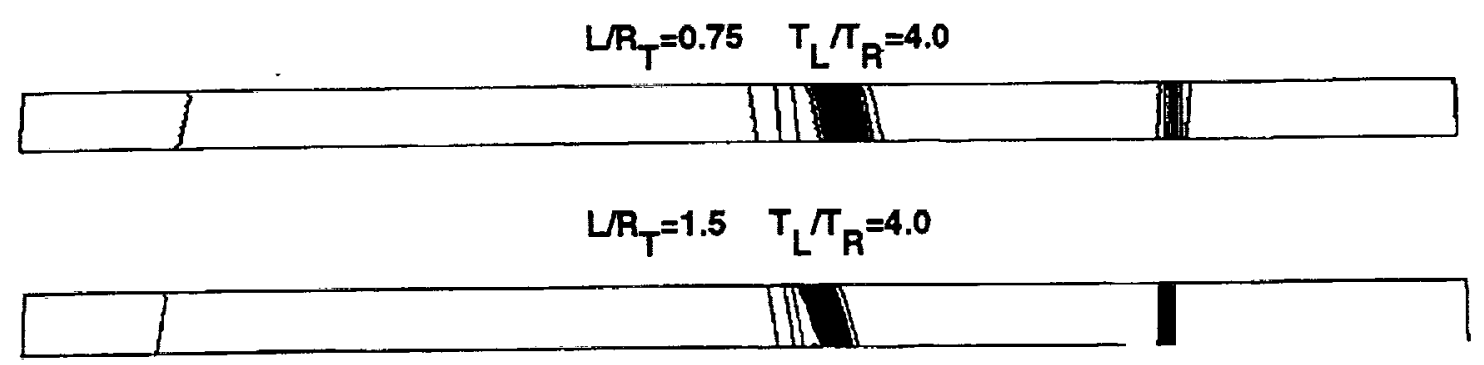

(a) $\mathrm{t}=0.25$

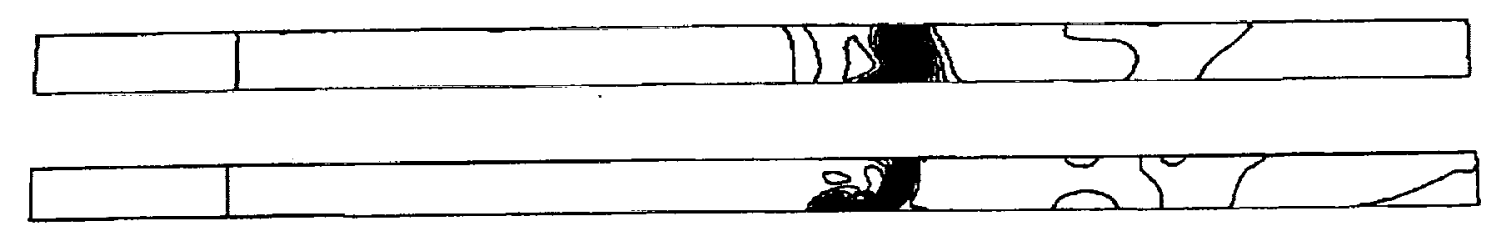

(b) $t=1.0$

Figure 13. Effect of varying length-to-tip radius ratio for $T_{L} \pi_{R}=4.0$ and $P_{L} / P_{R}=2.0$. 


$$
R_{H} / R_{T}=0.934 \quad T_{L} T_{R}=1.0 \quad M o=0.5
$$

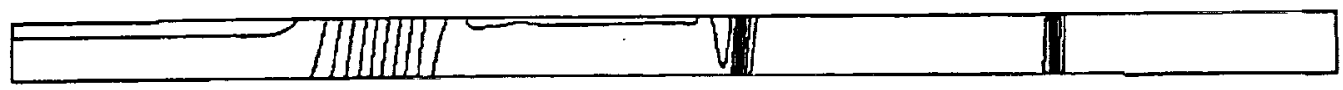

$$
R_{H} / R_{T}=0.5 \quad T_{L} / \pi_{R}=1.0 \quad M 0=0.5
$$

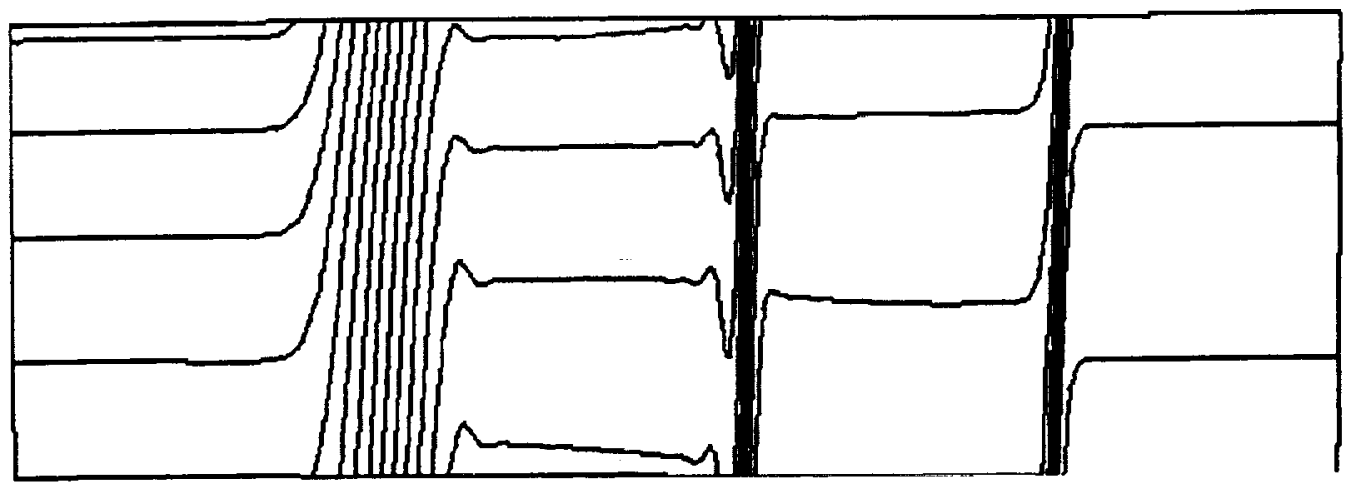

(a) $t=0.25$
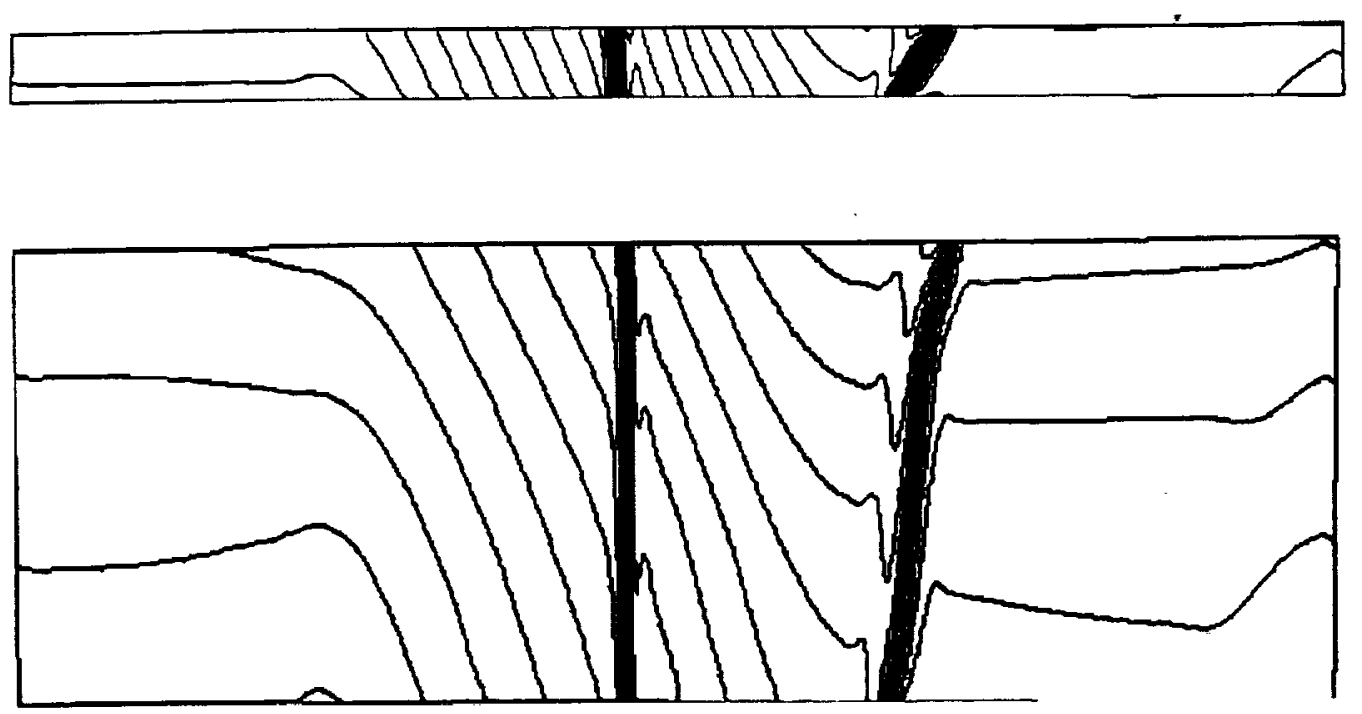

(b) $t=1.0$

Figure 14. Effect of varying hub-to-tip radius ratio for $T_{L} T_{R}=1.0$ and $P_{L} / P_{R}=2.0$. 


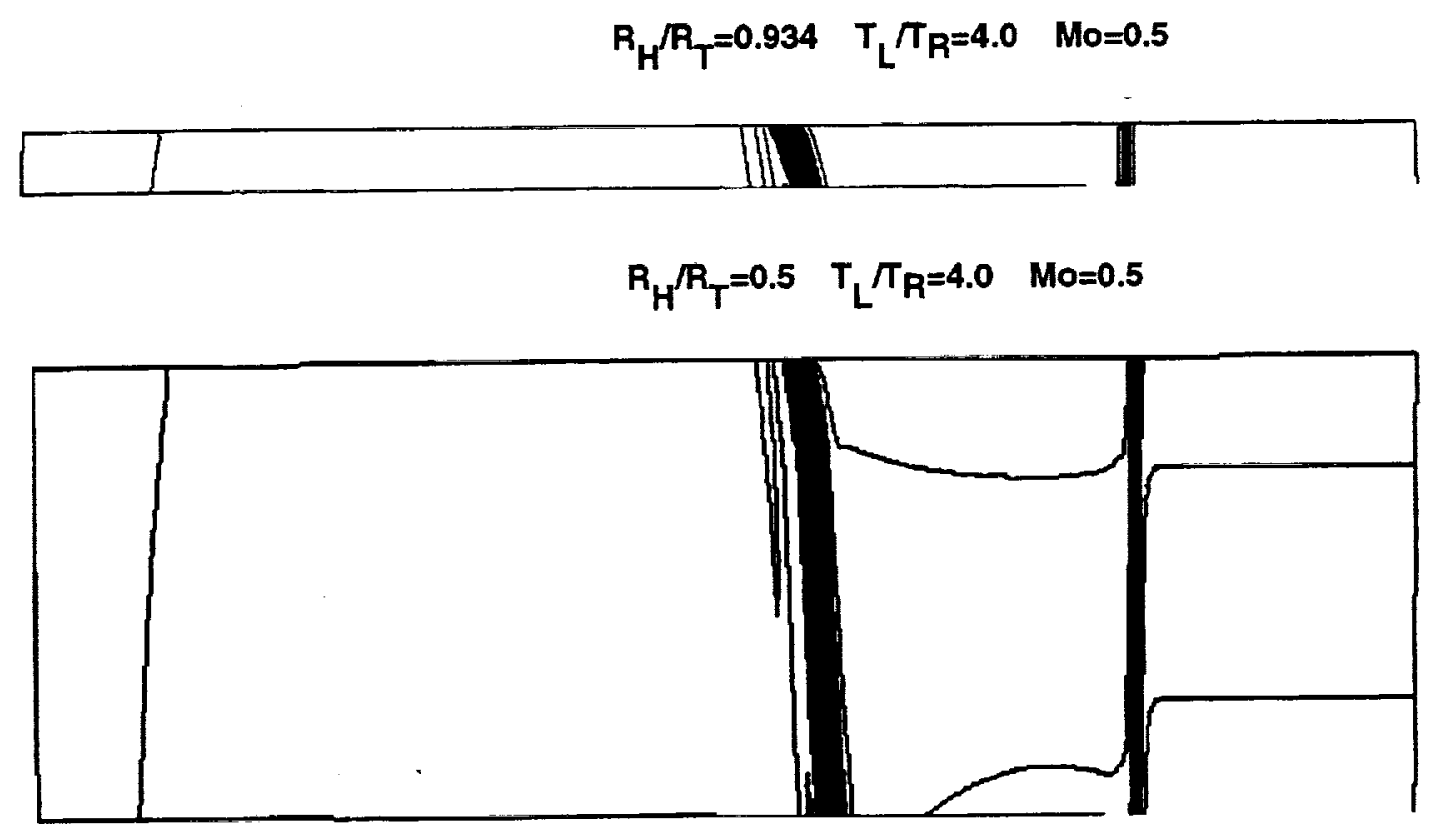

(a) $t=0.25$
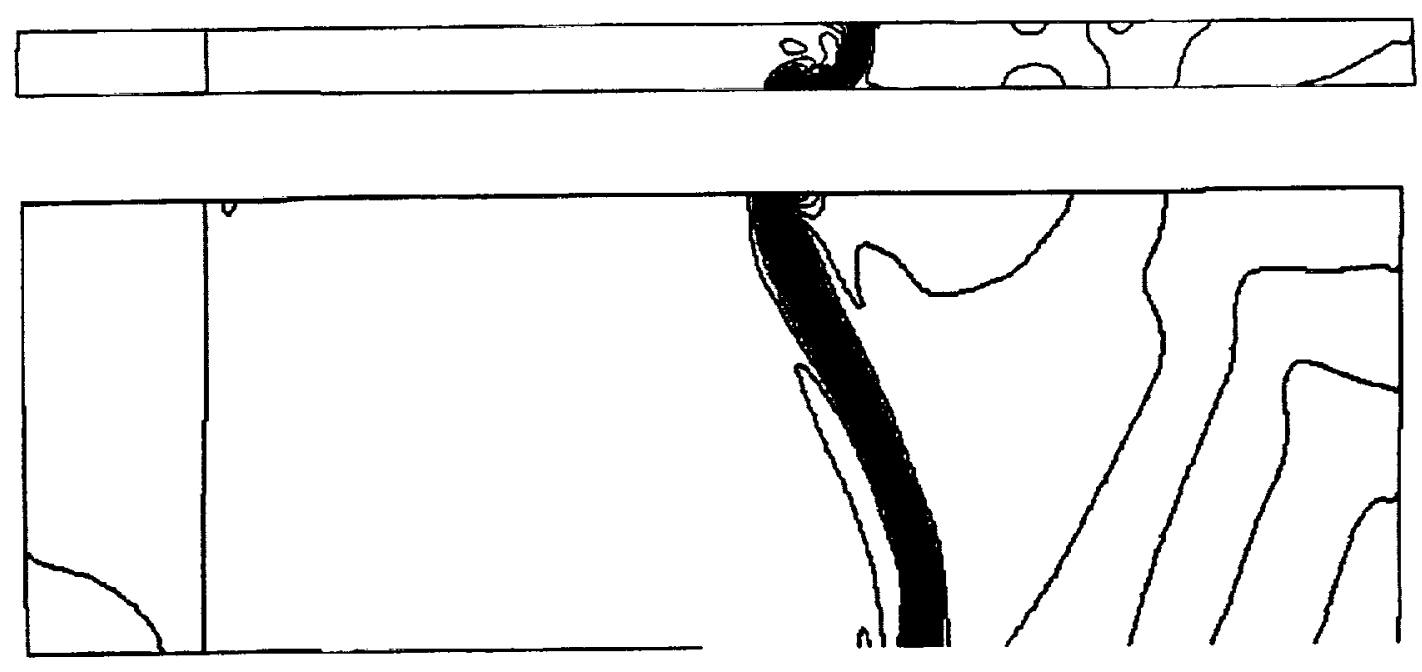

(b) $t=1.0$

Figure 15. Effect of varying hub-to-tip radius ratio for $T_{L} \pi_{R}=4.0$ and $P_{L} / P_{R}=2.0$. 
Public reporting burden for this collection of intormation is estimated to average 1 hour per response, including the time for reviewing instructions, searching existing data sources, Public reporting burdening the data needed, and completing and reviewing the collection of information. Send comments regarding this burden estimate or any other aspect of this collection of information, Including suggestions for reducing this burden, to Washington Headquarters Services, Directorate for Information Operations and Reports, 1215 Jelfor Davis

\begin{tabular}{l|l|l|} 
1. AGENCY USE ONLY (Leave blank) & 2. AEPOAT DATE & 3. REPOAT TYPE AND DATES COVERED
\end{tabular}

\begin{tabular}{|r|r|}
\hline June 1993 \\
\hline
\end{tabular}

Final Contractor Report

4. TITLE AND SUBTITLE

Analysis of Unsteady Wave Processes in a Rotating Channel

\section{FUNDING NUMBERS}

WU-505-62-10

NCC-208

\section{AUTHOR(S)}

L.M. Larosiliere and M. Mawid

\section{PERFORMING ORGANRATION NAME(S) AND ADDRESS(ES)}

8. PERforming organization REPORT NUMBER

Ohio Aerospace Institute 22800 Cedar Point Road

Brook Park, Ohio 44142

9. SPONSORING/MONITORING AGENCY NAME(S) AND ADDRESS(ES)

National Aeronautics and Space Administration

Lewis Research Center

Cleveland, Ohio 44135-3191
10. SPONSORINGMONTTORING AGENCY REPORT NUMBER

NASA CR-191154

AIAA-93-2527

\section{SUPPLEMENTARY NOTES}

Prepared for the 29th Joint Propulsion Conference and Exhibit cosponsored by the AIAA, SAE, ASME, and ASEE, Monterey, California, June 28-30, 1993. L.M. Larosiliere, Ohio Aerospace Insitute, 22800 Brook Park Road, Brook Park, Ohio 44142; and M. Mawid, University of Illinois at Chicago, Chicago, Illinois 60680. Project Manager, Lawrence J. Bober, (216) 433-3944.

12. DISTRIBUTION/AVAILABILITY STATEMENT

12b. DISTRIBUTION CODE

Unclassified - Unlimited

Subject Category 07

\section{ABSTRACT (Maximum 200 words)}

The impact of passage rotation on the gasdynamic wave processes is analyzed through a numerical simulation of ideal shock-tube flow in a closed rotating-channel. Initial conditions are prescribed by assuming homentropic solid-body rotation. Relevant parameters of the problem such as wheel Mach number, hub-to-tip radius ratio, length-to-tip radius ratio, diaphragm temperature ratio, and diaphragm pressure ratio are varied. The results suggest possible criteria for assessing the consequences of passage rotation on the wave processes, and they may therefore be applicable to pressure-exchange wave rotors. It is shown that for a fixed geometry and initial conditions, the contact interface acquires a distorted three-dimensional time-dependent orientation at non-zero wheel Mach numbers. At a fixed wheel Mach number, the level of distortion depends primarily on the density ratio across the interface as well as the hub-to-tip radius ratio. Rarefaction fronts, shocks, and contact interfaces are observed to propagate faster with increasing wheel Mach number.

14. SUB.JECT TERMS

CFD; Unsteady waves; Centrifugal forces

15. NUMBER OF PAGES

17. SECUATY CLASSIFICATION
OF REPORT

Unclassified

18. SECURITY CLASSIFICATION
OF THIS PAGE
Unclassified

Unclassified
19. SECURITY CLASSIFICATION OF ABSTRACT Unclassified
20. LNITATION OF ABSTRACT

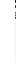

